\title{
A Gessel-Viennot-Type Method for Cycle Systems in a Directed Graph
}

\author{
Christopher R. H. Hanusa \\ Department of Mathematical Sciences \\ Binghamton University, Binghamton, New York, USA \\ chanusa@math. binghamton.edu
}

Submitted: Nov 28, 2005; Accepted: Mar 31, 2006; Published: Apr 4, 2006

Mathematics Subject Classifications: Primary 05B45, 05C30;

Secondary 05A15, 05B20, 05C38, 05C50, 05C70, 11A51, 11B83, 15A15, 15A36, 52C20

Keywords: directed graph, cycle system, path system, walk system, Aztec diamond,

Aztec pillow, Hamburger Theorem, Kasteleyn-Percus, Gessel-Viennot, Schröder numbers

\begin{abstract}
We introduce a new determinantal method to count cycle systems in a directed graph that generalizes Gessel and Viennot's determinantal method on path systems. The method gives new insight into the enumeration of domino tilings of Aztec diamonds, Aztec pillows, and related regions.
\end{abstract}

\section{Introduction}

In this article, we present an analogue of the Gessel-Viennot method for counting cycle systems on a type of directed graph we call a hamburger graph. A hamburger graph $H$ is made up of two acyclic graphs $G_{1}$ and $G_{2}$ and a connecting edge set $E_{3}$ with the following properties. The graph $G_{1}$ has $k$ distinguished vertices $\left\{v_{1}, \ldots, v_{k}\right\}$ with directed paths from $v_{i}$ to $v_{j}$ only if $i<j$. The graph $G_{2}$ has $k$ distinguished vertices $\left\{w_{k+1}, \ldots, w_{2 k}\right\}$ with directed paths from $w_{i}$ to $w_{j}$ only if $i>j$. The edge set $E_{3}$ connects the vertices $v_{i}$ and $w_{k+i}$ by way of edges $e_{i}: v_{i} \rightarrow w_{k+i}$ and $e_{i}^{\prime}: w_{k+i} \rightarrow v_{i}$. (See Figure 1 for a visualization.) Hamburger graphs arise naturally in the study of Aztec diamonds, as explained in Section 5.

The Gessel-Viennot method is a determinantal method to count path systems in an acyclic directed graph $G$ with $k$ sources $s_{1}, \ldots, s_{k}$ and $k$ sinks $t_{1}, \ldots, t_{k}$. A path system $\mathcal{P}$ is a collection of $k$ vertex-disjoint paths, each one directed from $s_{i}$ to $t_{\sigma(i)}$, for some permutation $\sigma \in S_{k}$ (where $S_{k}$ is the symmetric group on $k$ elements). Call a path system $\mathcal{P}$ positive if the sign of this permutation $\sigma$ satisfies $\operatorname{sgn}(\sigma)=+1$ and negative if $\operatorname{sgn}(\sigma)=-1$. Let $p^{+}$be the number of positive path systems and $p^{-}$be the number of negative path systems. 


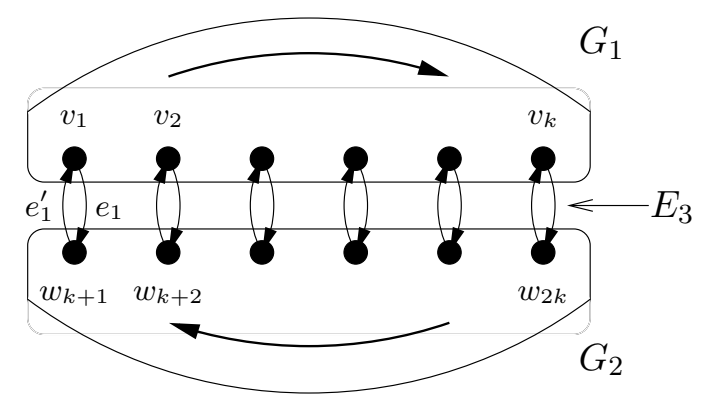

Figure 1: A hamburger graph

Corresponding to this graph $G$ is a $k \times k$ matrix $A=\left(a_{i j}\right)$, where $a_{i j}$ is the number of paths from $s_{i}$ to $t_{j}$ in $G$. The result of Gessel and Viennot states that $\operatorname{det} A=p^{+}-p^{-}$. The Gessel-Viennot method was introduced in $[4,5]$, and has its roots in works by Karlin and McGregor [8] and Lindström [10]. A nice exposition of the method and applications is given in the article by Aigner [1].

This article concerns a similar determinantal method for counting cycle systems in a hamburger graph $H$. A cycle system $\mathcal{C}$ is a collection of vertex-disjoint directed cycles in $H$. Let $l$ be the number of edges in $\mathcal{C}$ that travel from $G_{2}$ to $G_{1}$ and let $m$ be the number of cycles in $\mathcal{C}$. Call a cycle system positive if $(-1)^{l+m}=+1$ and negative if $(-1)^{l+m}=-1$. Let $c^{+}$be the number of positive cycle systems and $c^{-}$be the number of negative cycle systems. Corresponding to each hamburger graph $H$ is a $2 k \times 2 k$ block matrix $M_{H}$ of the form

$$
M_{H}=\left[\begin{array}{cc}
A & I_{k} \\
-I_{k} & B
\end{array}\right],
$$

where in the upper triangular matrix $A=\left(a_{i j}\right), a_{i j}$ is the number of paths from $v_{i}$ to $v_{j}$ in $G_{1}$ and in the lower triangular matrix $B=\left(b_{i j}\right), b_{i j}$ is the number of paths from $w_{k+i}$ to $w_{k+j}$ in $G_{2}$. This matrix $M_{H}$ is referred to as a hamburger matrix.

Theorem 1.1 (The Hamburger Theorem). If $H$ is a hamburger graph, then $\operatorname{det} M_{H}=$ $c^{+}-c^{-}$.

A hamburger graph $H$ is called strongly planar if there is a planar embedding of $H$ that sends $v_{i}$ to $(i, 1)$ and $w_{k+i}$ to $(i,-1)$ for all $1 \leq i \leq k$, and keeps edges of $E_{1}$ in the half-space $y \geq 1$ and edges of $E_{2}$ in the half-space $y \leq-1$. This definition suggests that $G_{1}$ and $G_{2}$ are "relatively" planar in $H$, a stronger condition than planarity of $H$. Notice that when $H$ is strongly planar, each cycle must use exactly one edge from $G_{2}$ to $G_{1}$. Hence, the sign of every cycle system is +1 . This implies the following corollary.

Corollary 1.2. If $H$ is a strongly planar hamburger graph, $\operatorname{det} M_{H}=c^{+}$.

The following simple example serves to guide us. Consider the two graphs $G_{1}=$ $\left(V_{1}, E_{1}\right)$ and $G_{2}=\left(V_{2}, E_{2}\right)$, where $V_{1}=\left\{v_{1}, v_{2}, v_{3}, v\right\}, V_{2}=\left\{w_{4}, w_{5}, w_{6}, w\right\}, E_{1}=\left\{v_{1} \rightarrow\right.$ $\left.v_{2}, v_{2} \rightarrow v_{3}, v_{1} \rightarrow v, v \rightarrow v_{3}\right\}$, and $E_{2}=\left\{w_{6} \rightarrow w_{5}, w_{5} \rightarrow w_{4}, w_{6} \rightarrow w, w \rightarrow w_{4}\right\}$. Our 


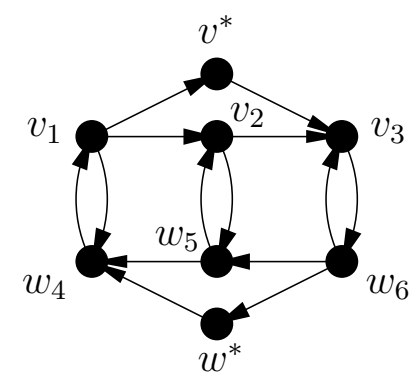

Figure 2: A simple hamburger graph $H$

hamburger graph $H$ will be the union of $G_{1}, G_{2}$, and the edge set $E_{3}$. In this example, $k=3$ and $H$ is strongly planar. Figure 2 gives a graphical representation of $H$.

In this example, the hamburger matrix $M_{H}$ equals

$$
M_{H}=\left[\begin{array}{cccccc}
1 & 1 & 2 & 1 & 0 & 0 \\
0 & 1 & 1 & 0 & 1 & 0 \\
0 & 0 & 1 & 0 & 0 & 1 \\
-1 & 0 & 0 & 1 & 0 & 0 \\
0 & -1 & 0 & 1 & 1 & 0 \\
0 & 0 & -1 & 2 & 1 & 1
\end{array}\right]
$$

The determinant of $M_{H}$ is 17 , corresponding to the seventeen cycle systems (each with sign +1 ) in Figure 3.

The graph that inspired the definition of a hamburger graph comes from the work of Brualdi and Kirkland [2], in which they give a new proof that the number of domino tilings of the Aztec diamond is $2^{n(n+1) / 2}$. An Aztec diamond, denoted by $A D_{n}$, is the union of the $2 n(n+1)$ unit squares with integral vertices $(x, y)$ such that $|x|+|y| \leq n+1$. See Figure 4 for an example of an Aztec diamond, as well as an example of an Aztec pillow and a generalized Aztec pillow, described in the next paragraphs.

An Aztec pillow, as it was initially presented in [12], is also a rotationally symmetric region in the plane. On the top left boundary, however, the steps are composed of three squares to the right for every square up. Another definition is that Aztec pillows are the union of the unit squares with integral vertices $(x, y)$ such that $|x+y|<n+1$ and $|3 y-x|<n+3$. As with Aztec diamonds, we denote the Aztec pillow with $2 n$ squares in each of the central rows by $A P_{n}$. In Section 6, we extend the notion of Aztec pillows having steps of length 3 to "odd pillows" - those that have steps that are of a constant odd length. The integral vertices $(x, y)$ of the unit squares in $q$-pillows for $q$ odd satisfy $|x+y|<n+1$ and $|q y-x|<n+q$.

We introduce the idea of a generalized Aztec pillow, where the steps on all diagonals are of possibly different odd lengths. More specifically, a generalized Aztec pillow is a horizontally convex and vertically convex region such that the steps both up and down in each diagonal have an odd number of squares horizontally for every one square vertically. 

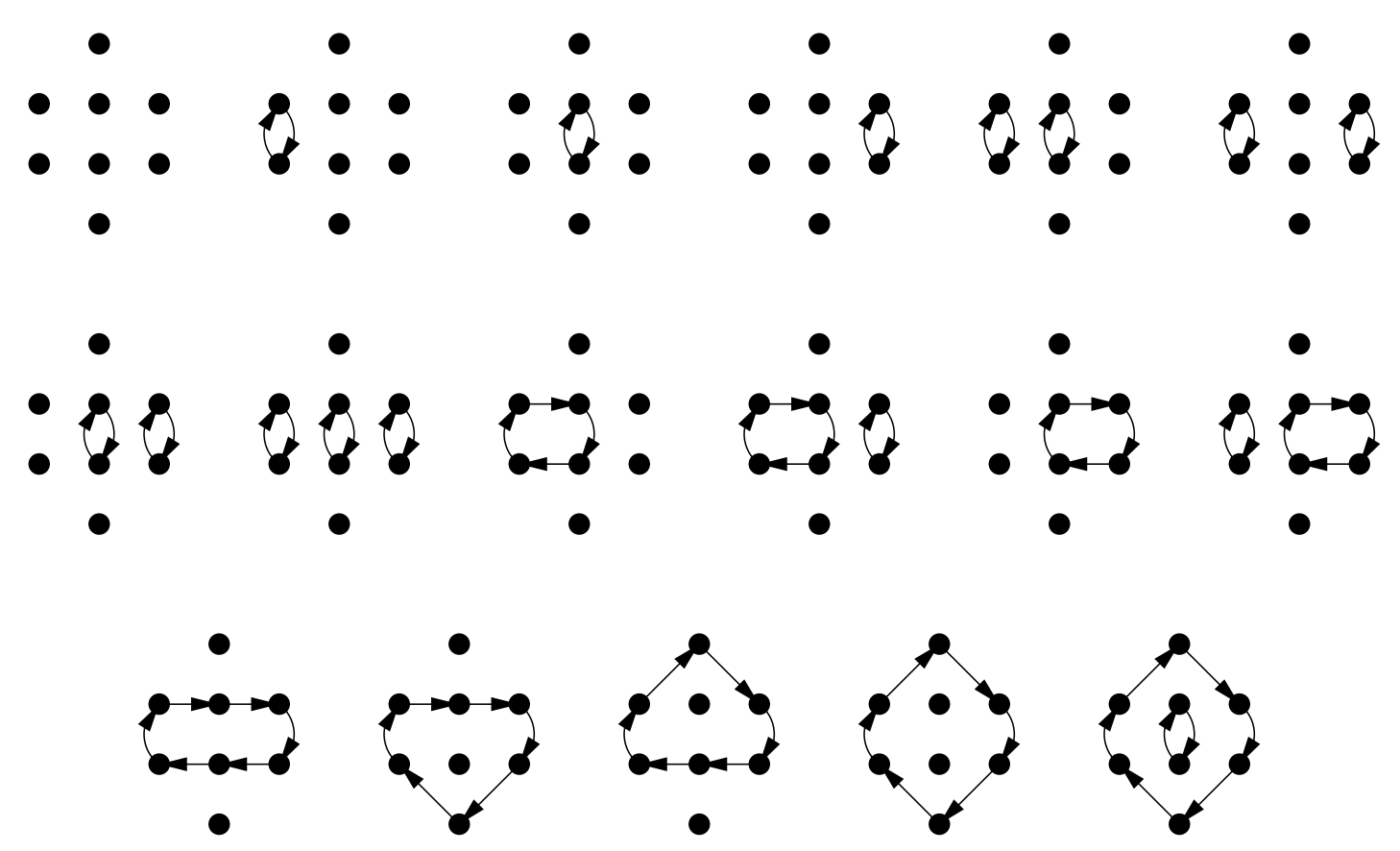

Figure 3: The seventeen cycle systems for the hamburger graph in Figure 2
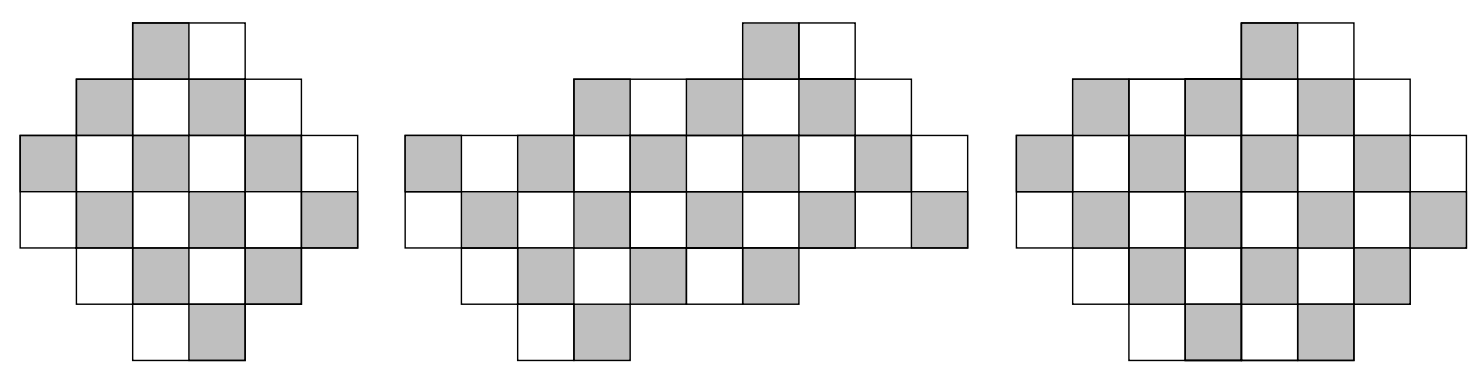

Figure 4: Examples of an Aztec diamond, an Aztec pillow, and a generalized Aztec pillow 
A key fact that we will use is that any generalized Aztec pillow can be recovered from a large enough Aztec diamond by the placement of horizontal dominoes.

Brualdi and Kirkland prove the formula for the number of domino tilings of an Aztec diamond by creating an associated digraph and counting its cycle systems, manipulating the digraph's associated Kasteleyn-Percus matrix of order $n(n+1)$. To learn about Kasteleyn theory and Kasteleyn-Percus matrices, start with Kasteleyn's 1961 work [9] and Percus's 1963 work [11]. The Hamburger Theorem proves that we can count the number of domino tilings of an Aztec diamond with a much smaller determinant, of order $2 n$. A Schur complement allows us to reduce the determinant calculation to one of order $n$. An analogous reduction in determinant size (from order $O\left(n^{2}\right)$ to order $O(n)$ ) occurs for all regions to which this theorem applies, including generalized Aztec pillows. In addition, whereas Kasteleyn theory applies only to planar graphs, there is no planarity restriction for hamburger graphs. For this reason, the Hamburger Theorem gives a new counting method for cycle systems in some non-planar graphs.

More recently, $\mathrm{Eu}$ and $\mathrm{Fu}$ present a new proof of the number of tilings of an Aztec diamond [3]. Their lattice-path-based proof also reduces to an $n \times n$ determinant but does not generalize to the case of Aztec pillows. This result is discussed further in Section 5.2.

In Section 2, we present an overview of the proof of the Hamburger Theorem, including the key lemmas involved. The necessary machinery is built up in Section 3 to complete the proof in Section 4. Section 5 presents applications of the Hamburger Theorem to Aztec diamonds, Aztec pillows, and generalized Aztec pillows. Section 6 concludes with a counterexample to the most natural generalization of the Hamburger Theorem and an extension of Propp's Conjecture on Aztec pillows.

\section{Outline of the Proof of the Hamburger Theorem}

\subsection{The Hamburger Theorem}

Like the proof of the Gessel-Viennot method, the proof of the Hamburger Theorem hinges on cancellation of terms in the permutation expansion of the determinant of $M_{H}$. In the proof, we must allow closed directed walks in addition to cycles. We must also allow walk systems, arbitrary collections of closed directed walks, since they can and will appear in the permutation expansion of the hamburger determinant. We call a walk system simple if the set of walks visits no vertex more than once. We call a cycle of the form $c: v_{i} \rightarrow w_{i+k} \rightarrow v_{i}$ a 2-cycle.

Each signed term in the permutation expansion of the hamburger determinant is the contribution of many signed walk systems $\mathcal{W}$. Walk systems that are not cycle systems will all cancel out in the determinant expansion. We will show this in two steps. We start by considering walk systems that are not simple. If this is the case, one of the two following properties MAY hold.

Property 1. The walk system contains a walk that has a self-intersection.

Property 2. The walk system has two intersecting walks, neither of which is a 2-cycle. 
The following lemma shows that the contributions of walk systems satisfying either of these two properties cancel in the permutation expansion of the determinant of $M_{H}$.

Lemma 2.1. The set of all walk systems $\mathcal{W}$ that satisfy either Property 1 or Property 2 can be partitioned into equivalence classes, each of which contributes a net zero to the permutation expansion of the determinant of $M_{H}$.

The proof of Lemma 2.1 uses a generalized involution principle. Walk systems cancel in families based on the their "first" intersection point.

The remainder of the cancellation in the determinant expansion is based on the concept of a minimal walk system; we motivate this definition by asking the following questions. What kind of walk systems does the permutation expansion of the hamburger determinant generate, and how is this different from our original notion of cycle systems that we wanted to count in the introduction? The key difference is that the same collection of walks can be generated by multiple terms in the determinantal expansion of $M_{H}$; whereas, we would only want to count it once as a cycle system. This redundancy arises when the walk visits three distinguished vertices in $G_{1}$ without passing via $G_{2}$ or vice versa. We illustrate this notion with the following example.

Consider the second cycle system in the third row of Figure 3, consisting of one solitary directed cycle. Since this cycle visits vertices $v_{1}, v_{2}, v_{3}, w_{6}$, and $w_{4}$ in that order, it contributes a non-zero weight in the permutation expansion of the determinant corresponding to the term (12364) in $S_{6}$. Notice that this cycle also contributes a nonzero weight in the permutation expansion of the determinant corresponding to the term (1364). We see this since our cycle follows a path from $v_{1}$ to $v_{3}$ (by way of $v_{2}$ ), returning to $v_{1}$ via $w_{6}$ and $w_{4}$. We must deal with this ambiguity. We introduce the idea of a minimal permutation cycle, one which does not include more than two successive entries with values between 1 and $k$ or between $k+1$ and $2 k$. We see that (1364) is minimal while (12364) is not.

We notice that walk systems arise from permutations, so it is natural to think of a walk system as a permutation together with a collection of walks that "follow" the permutation. This is the idea of a walk system-permutation pair (or WSP-pair for short) that is presented in Section 3.4. From the idea of a minimal permutation cycle, we define a minimal walk to have as its base permutation a minimal permutation cycle, and a minimal walk system to be composed of only minimal walks. Since our original goal was to count "cycle systems" in a directed graph, we realize we need to be precise and instead count "simple minimal walk systems". This leads to the second part of the proof of the Hamburger Theorem.

Given a walk system that is either not simple or not minimal and that satisfies neither Property 1 nor Property 2, at least one of the two following properties MUST hold.

Property 3. The walk system has two intersecting walks, one of which is a 2-cycle.

Property 4. The walk system is not minimal.

The following lemma shows that the contributions of walk systems satisfying either of these new properties cancel in the permutation expansion of the determinant of $M_{H}$. 
Lemma 2.2. The set of all walk systems $\mathcal{W}$ that satisfy neither Property 1 nor Property 2 and that satisfy Property 3 or Property 4 can be partitioned into equivalence classes, each of which contributes a net zero to the permutation expansion of the determinant of $M_{H}$.

The proof of Lemma 2.2 is also based on involutions. Walk systems cancel in families built from an index set containing the set of all 2-cycle intersections and non-minimalities.

If a walk system satisfies none of the conditions of Properties 1 through 4, then it is indeed a simple minimal walk system, or in other words, a cycle system. The cancellation from the above sets of families gives that only cycle systems contribute to the permutation expansion of the determinant of $M_{H}$. This contribution is the signed weight of each cycle system, so the determinant of $M_{H}$ exactly equals $c^{+}-c^{-}$. Theorem 1.1 follows from Lemmas 2.1 and 2.2 in Section 4.

\subsection{The Weighted Hamburger Theorem}

There is also a weighted version of the Hamburger Theorem, and it will be under this generalization that Lemmas 2.1 and 2.2 are proved. We allow weights $\mathrm{wt}(e)$ on the edges of the hamburger graph; the simplest weighting, which counts the number of cycle systems, assigns $\operatorname{wt}(e) \equiv 1$. We require that $\operatorname{wt}\left(e_{i}\right) \operatorname{wt}\left(e_{i}^{\prime}\right)=1$ for all $2 \leq i \leq k-1$, but we do not require this condition for $i=1$ nor for $i=k$. Define the $2 k \times 2 k$ weighted hamburger matrix $M_{H}$ to be the block matrix

$$
M_{H}=\left[\begin{array}{cc}
A & D_{1} \\
-D_{2} & B
\end{array}\right] .
$$

In the upper-triangular $k \times k$ matrix $A=\left(a_{i j}\right), a_{i j}$ is the sum of the products of the weights of edges over all paths from $v_{i}$ to $v_{j}$ in $G_{1}$. In the lower triangular $k \times k$ matrix $B=\left(b_{i j}\right), b_{i j}$ is the sum of the products of the weights of edges over all paths from $w_{k+i}$ to $w_{k+j}$ in $G_{2}$. The diagonal $k \times k$ matrix $D_{1}$ has as its entries $d_{i i}=\operatorname{wt}\left(e_{i}\right)$ and the diagonal $k \times k$ matrix $D_{2}$ has as its entries $d_{i i}=\operatorname{wt}\left(e_{i}^{\prime}\right)$. Note that when the weights of the edges in $E_{3}$ are all 1 , these matrices satisfy $D_{1}=D_{2}=I_{k}$.

We wish to count vertex-disjoint unions of weighted cycles in $H$. In any hamburger graph $H$, there are two possible types of cycle. There are $k 2$-cycles

$$
c: v_{i} \stackrel{e_{i}}{\longrightarrow} w_{k+i} \stackrel{e_{i}^{\prime}}{\longrightarrow} v_{i}
$$

and many more general cycles that alternate between $G_{1}$ and $G_{2}$. We can think of a general cycle as a path $P_{1}$ in $G_{1}$ connected by an edge $e_{1,1} \in E_{3}$ to a path $Q_{1}$ in $G_{2}$, which in turn connects to a path $P_{2}$ in $G_{1}$ by an edge $e_{1,2}^{\prime}$, continuing in this fashion until arriving at a final path $Q_{l}$ in $G_{2}$ whose terminal vertex is adjacent to the initial vertex of $P_{1}$. We write

$$
c: P_{1} \stackrel{e_{1,1}}{\longrightarrow} Q_{1} \stackrel{e_{1,2}^{\prime}}{\longrightarrow} P_{2} \stackrel{e_{2,1}}{\longrightarrow} \cdots \stackrel{e_{l, 1}}{\longrightarrow} P_{l} \stackrel{e_{l, 2}^{\prime}}{\longrightarrow} Q_{l} .
$$


For each cycle $c$, we define the weight $\operatorname{wt}(c)$ of $c$ to be the product of the weights of all edges traversed by $c$ :

$$
\mathrm{wt}(c)=\prod_{e \in c} \mathrm{wt}(e)
$$

We define a weighted cycle system to be a collection $\mathcal{C}$ of $m$ vertex-disjoint cycles. We again define the sign of a weighted cycle system to be $\operatorname{sgn}(\mathcal{C})=(-1)^{l+m}$, where $l$ is the total number of edges from $G_{2}$ to $G_{1}$ in $\mathcal{C}$. We say that a weighted cycle system $\mathcal{C}$ is positive if $\operatorname{sgn}(\mathcal{C})=+1$ and negative if $\operatorname{sgn}(\mathcal{C})=-1$. For a hamburger graph $H$, let $c^{+}$be the sum of the weights of positive weighted cycle systems, and let $c^{-}$be the sum of the weights of negative weighted cycle systems.

Theorem 2.3 (The weighted Hamburger Theorem). The determinant of the weighted hamburger matrix $M_{H}$ equals $c^{+}-c^{-}$.

As above, Theorem 2.3 follows from Lemmas 2.1 and 2.2. The proofs will be presented after developing the following necessary machinery.

\section{Additional Definitions}

\subsection{Edge Cycles and Permutation Cycles}

In the proof of the Hamburger Theorem, there are two distinct mathematical objects that have the name "cycle". We have already mentioned the type of cycle that appears in graph theory. There, a (simple) cycle in a directed graph is a closed directed path with no repeated vertices.

Secondly, there is a notion of cycle when we talk about permutations. If $\sigma \in S_{n}$ is a permutation, we can write $\sigma$ as the product of disjoint cycles $\sigma=\chi_{1} \chi_{2} \cdots \chi_{\tau}$.

To distinguish between these two types of cycles when confusion is possible, we call the former kind an edge cycle and the latter kind a permutation cycle. Notationally, we use Roman letters when discussing edge cycles and Greek letters when discussing permutation cycles.

\subsection{Permutation Expansion of the Determinant}

We recall that the permutation expansion of the determinant of an $n \times n$ matrix $M=\left(m_{i j}\right)$ is the expansion of the determinant as

$$
\operatorname{det} M=\sum_{\sigma \in S_{n}}(\operatorname{sgn} \sigma) m_{1, \sigma(1)} \cdots m_{n, \sigma(n)} .
$$

We will be considering non-zero terms in the permutation expansion of the determinant of the hamburger matrix $M_{H}$. Because of the special block form of the hamburger matrix in Equation (1), the permutations $\sigma$ that make non-zero contributions to this sum are products of disjoint cycles of either of two forms - the simple transposition

$$
\chi=\left(\varphi_{11} \omega_{11}\right)
$$


or the general permutation cycle

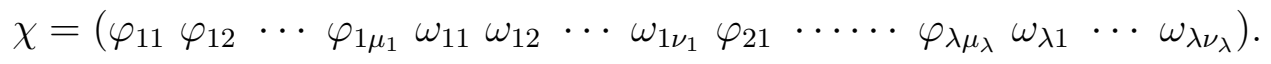

In the first case, $\omega_{11}=\varphi_{11}+k$. In the second case, $1 \leq \varphi_{\iota \kappa} \leq k, k+1 \leq \omega_{\iota \kappa} \leq 2 k$, $\varphi_{\iota \kappa}<\varphi_{\iota, \kappa+1}$, and $\omega_{\iota \kappa}>\omega_{\iota, \kappa+1}$ for all $1 \leq \iota \leq \lambda$ and relevant $\kappa$. The block matrix form also implies that $\varphi_{\iota \mu_{\iota}}+k=\omega_{\iota 1}, \omega_{\iota \nu_{\iota}}-k=\varphi_{\iota+1,1}$, and $\omega_{\lambda \nu_{\lambda}}-k=\varphi_{11}$. These last requirements along with the fact that no integers appear more than once in a permutation cycle imply that $\mu_{i}, \nu_{i} \geq 2$ for $1 \leq i \leq \lambda$. So that this permutation cycle is in standard form, we make sure that $\varphi_{11}=\min _{\iota, \kappa} \varphi_{\iota \kappa}$. In order to refer to this value later, we define a function $\Phi$ by $\Phi(\chi)=\varphi_{11}$. Each value $1 \leq \varphi_{\iota} \leq k$ or $k+1 \leq \omega_{\iota} \leq 2 k$ appears at most once for any $\sigma \in S_{2 k}$.

We call a permutation cycle $\chi$ minimal if it is a transposition or if $\mu_{\iota}=\nu_{\iota}=2$ for all $\iota$. Minimality implies that we can write our general permutation cycles $\chi$ in the form

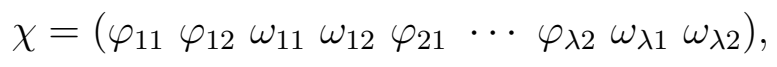

with the same conditions as before. We call a permutation $\sigma=\chi_{1} \cdots \chi_{\tau}$ minimal if each of its cycles $\chi_{\iota}$ is minimal.

\subsection{Walks Associated to a Permutation}

To each permutation cycle $\chi \in S_{2 k}$, we can associate one or more walks $c_{\chi}$ in $H$.

If $\chi$ is the transposition $\chi=\left(\varphi_{11} \omega_{11}\right)$, then we associate the 2-cycle $c_{\chi}: v_{\varphi_{11}} \rightarrow$ $w_{\omega_{11}} \rightarrow v_{\varphi_{11}}$ to $\chi$. To any permutation cycle $\chi$ that is not a transposition, we can associate multiple walks $c_{\chi}$ by gluing together paths that follow $\chi$ in the following way. If $\chi$ has the form of Equation (3), then for each $1 \leq i \leq \lambda$, let $P_{i}$ be any path in $G_{1}$ that visits each of the vertices $v_{\varphi_{i 1}}, v_{\varphi_{i 2}}$, all the way through $v_{\varphi_{i \mu_{i}}}$ in order. Similarly, let $Q_{i}$ be any path in $G_{2}$ that visits each of the vertices $w_{\omega_{i 1}}, w_{\omega_{i 2}}$, through $w_{\omega_{i \nu_{i}}}$ in order. For each choice of paths $P_{i}$ and $Q_{i}$, we have a possibility for the walk $c_{\chi}$; we can set

$$
c_{\chi}: P_{1} \stackrel{e_{\varphi_{12}}}{\longrightarrow} Q_{1} \stackrel{e_{\varphi_{21}}^{\prime}}{\longrightarrow} P_{2} \stackrel{e_{\varphi_{22}}}{\longrightarrow} \cdots \cdots \stackrel{e_{\varphi_{\lambda 1}}^{\prime}}{\longrightarrow} P_{\lambda} \stackrel{e_{\varphi_{\lambda 2}}}{\longrightarrow} Q_{\lambda} .
$$

See Figure 5 for the choices of $c_{(12364)}$ in the hamburger graph presented in Figure 2. We call $\lambda$ the number of $P$-paths in $c_{\chi}$. The function $\Phi$, defined in the previous section, defines a partial ordering on walks in a walk system - we say that the associated walk $c_{\chi}$ comes before the associated walk $c_{\chi^{\prime}}$ if $\Phi(\chi)<\Phi\left(\chi^{\prime}\right)$. We call this the initial term order. As in Section 2.2, we define the weight of a walk $c_{\chi}$ to be the product of the weights of all edges traversed by $c_{\chi}$.

\subsection{Walk System-Permutation Pairs}

We defined walk systems in Section 2, but we will see that the proof of Theorem 1.1 requires us to think of walk systems first as a permutation and second as a collection of 


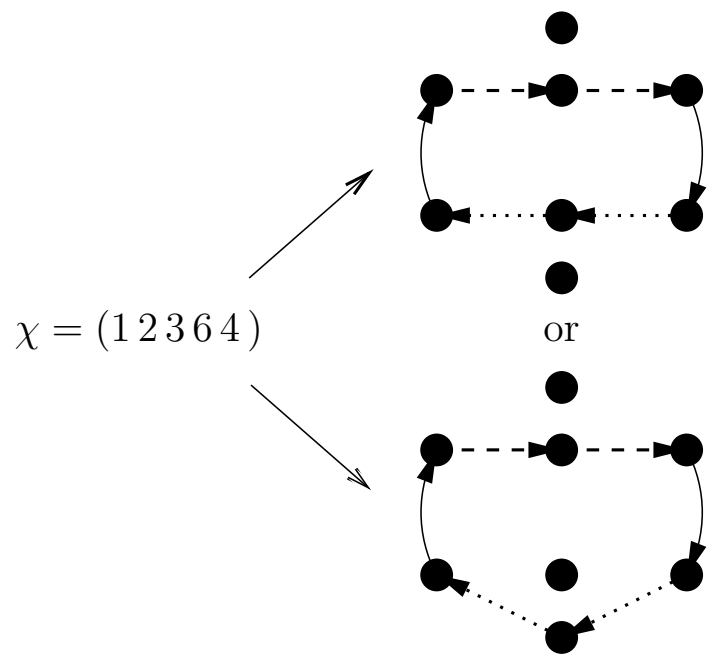

Figure 5: A permutation cycle $\chi$ and the two walks in $H$ associated to $\chi$

walks determined by the permutation. We will see that for cycle systems as presented initially, signs and weights are not changed by this recharacterization.

If $H$ is a hamburger graph with $k$ pairs of distinguished vertices, we define a walk system-permutation pair as follows.

Definition 3.1. A walk system-permutation pair (or WSP-pair for short) is a pair $(\mathcal{W}, \sigma)$, where $\sigma \in S_{2 k}$ is a permutation and $\mathcal{W}$ is a collection of walks $c \in \mathcal{W}$ with the following property: if the disjoint cycle representation of $\sigma$ is $\sigma=\chi_{1} \cdots \chi_{\tau}$, then $\mathcal{W}$ is a collection of $\tau$ walks $c_{\chi_{\iota}}$, for $1 \leq \iota \leq \tau$, where $c_{\chi_{\iota}}$ is a walk associated to the permutation cycle $\chi_{\iota}$.

We define the weight of a WSP-pair $(\mathcal{W}, \sigma)$ to be the product of the weights of the associated walks $c_{\chi} \in \mathcal{W}$.

Each permutation $\sigma$ yields many collections of walks $\mathcal{W}$, collections of walks $\mathcal{W}$ may be associated to many permutations $\sigma$, but any walk system $\mathcal{W}$ corresponds to one and only one minimal permutation $\sigma_{m}$. This is because, given any path as in Equation (5), we can read off the initial and terminal vertices of each $P_{i}$ and $Q_{i}$ in order, producing a well-defined permutation cycle $\sigma_{m}$. We define a WSP-pair $(\mathcal{W}, \sigma)$ to be minimal if $\sigma$ is a minimal permutation.

For a WSP-pair $(\mathcal{W}, \sigma)$, where $\sigma=\chi_{1} \cdots \chi_{\tau}$, we define the sign of the WSP-pair, $\operatorname{sgn}(\mathcal{W}, \sigma)$, to be $(-1)^{l} \operatorname{sgn}(\sigma)$, where $\lambda_{\chi}$ is the number of $P$-paths in $c_{\chi}$ and where $l=$ $\sum_{c_{\chi} \in \mathcal{W}} \lambda_{\chi}$. Alternatively, we could consider the $\operatorname{sign}$ of $(\mathcal{W}, \sigma)$ to be the product of the signs of its associated walks $c_{\chi}$, where the $\operatorname{sign}$ of $c_{\chi}$ is $\operatorname{sgn}\left(c_{\chi}\right)=(-1)^{\lambda_{\chi}} \operatorname{sgn}(\chi)$. We say that a WSP-pair $(\mathcal{W}, \sigma)$ is positive if $\operatorname{sgn}(\mathcal{W}, \sigma)=+1$ and is negative if $\operatorname{sgn}(\mathcal{W}, \sigma)=-1$.

Note that if $(\mathcal{W}, \sigma)$ is a minimal WSP-pair, then $\operatorname{sgn}\left(c_{\chi}\right)=+1$ for a transposition $\chi$ and $\operatorname{sgn}\left(c_{\chi}\right)=(-1)^{\lambda+1}$ if $\chi$ is of the form in Equation (4). In particular, when $(\mathcal{W}, \sigma)$ is minimal and simple, its sign and weight is consistent with the definition given in the introduction. 


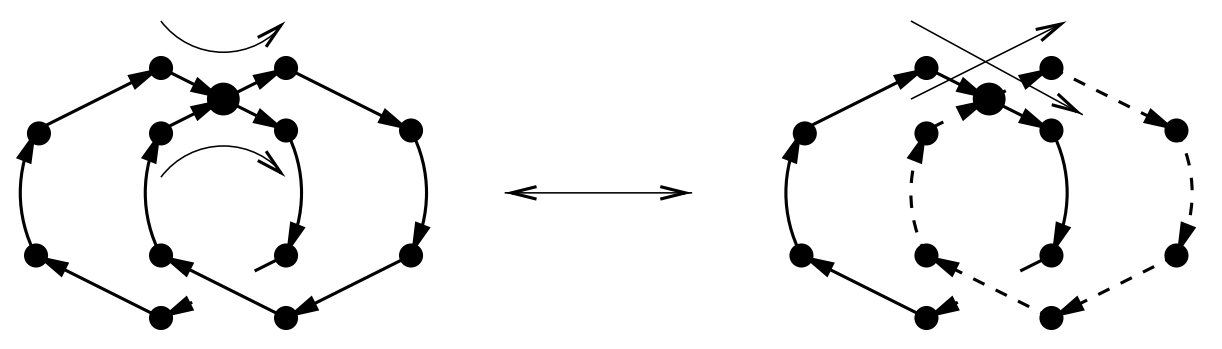

Figure 6: A self-intersecting cycle and its corresponding pair of intersecting cycles

\section{Proof of the Hamburger Theorem}

As mentioned in Section 2, we prove Lemmas 2.1 and 2.2 for the weighted version of the Hamburger Theorem, thereby proving Theorem 2.3. Theorem 1.1 follows as a special case of Theorem 2.3.

\subsection{Proof of Lemma 2.1, Part I}

Recall Properties 1 and 2 as well as Lemma 2.1.

Property 1. The walk system contains a walk that has a self-intersection.

Property 2. The walk system has two intersecting walks, neither of which is a 2-cycle.

Lemma 2.1. The set of all walk systems $\mathcal{W}$ that satisfy either Property 1 or Property 2 can be partitioned into equivalence classes, each of which contributes a net zero to the permutation expansion of the determinant of $M_{H}$.

The proof of Lemma 2.1 is a generalization of the involution principle, the idea of which comes from the picture presented in Figure 6. Given a self-intersecting walk, changing the order of edge traversal at the vertex of self-intersection leads to breaking the one self-intersecting walk into two walks that intersect at that same vertex. Since the edge set of the collection of walks has not changed, the weight of the two WSP-pairs is the same. We have introduced a transposition into the sign of the permutation cycle of the WSP-pair; this changes the sign of the WSP-pair, so these two WSP-pairs will cancel in the permutation expansion of the determinant of $M_{H}$.

One can imagine that this means that to every self-intersecting WSP-pair we can associate one WSP-pair with two cycles intersecting. However, more than one self-intersection may occur at this same point, and there may be additional walks that pass through that same point. Exactly what this WSP-pair would cancel with is not clear. If we decide to break all the self-intersections so that we have some number $N$ of walks through our vertex, it is not clear how we should sew the cycles back together. One starts to get the idea that we must consider all possible ways of sewing back together. Once we do just that, we have a family $\mathcal{F}$ of WSP-pairs, all of the same weight, whose net contribution to the permutation expansion of the determinant is zero.

This idea is conceptually simple but the proof is notationally complicated. 
If $\mathcal{W}$ satisfies either Property 1 or Property 2, then there is some vertex of intersection, be it either a self-intersection or an intersection of two walks. Our aim is to choose a welldefined first point of intersection at which we will build the family $\mathcal{F}$. The initial term order gives an order on walks associated to permutation cycles; we choose the earliest walk $c_{\chi_{\alpha}}$ that has some vertex of intersection. Once we have determined the earliest walk, we start at $v_{\Phi\left(c_{\chi_{\alpha}}\right)}$ and follow the walk

$$
c_{\chi_{a}}: P_{1} \rightarrow Q_{1} \rightarrow P_{2} \rightarrow \cdots \rightarrow P_{m} \rightarrow Q_{m}
$$

until we reach a vertex of intersection.

In our discussion, we make the assumption that this first vertex of intersection is a vertex $v^{*}$ in $G_{1}$. A similar argument exists if the first appearance occurs in $G_{2}$. Notice that at $v^{*}$ there may be multiple self-intersections or multiple intersections of walks. We will create a family $\mathcal{F}$ of WSP-pairs that takes into account each of these possibilities.

If we want to rigorously define the breaking of a self-intersecting walk at a vertex of self-intersection, we need to specify many different components of the WSP-pair $(C, \sigma)$. First, we need to specify on which walk in $\mathcal{W}$ we are acting. Next, we need to specify the vertex of self-intersection. Since this self-intersection vertex may occur in multiple paths, we need to specify which two paths we interchange in the breaking process.

\subsection{Definitions of Breaking and Sewing}

In the following paragraphs, we define "breaking" on WSP-pairs, which takes in a WSPpair $(\mathcal{W}, \sigma)$, one of $\sigma$ 's permutation cycles $\chi_{\alpha}$, the associated walk $c_{\chi_{\alpha}}$, paths $P_{y}$ and $P_{z}$ in $c_{\chi_{\alpha}}$, and the vertex $v^{*}$ in both $P_{y}$ and $P_{z}$ where $c_{\chi_{\alpha}}$ has a self-intersection. For simplicity, we assume that $v^{*}$ is not a distinguished vertex, but the argument still holds in that case. The inverse of this operation is "sewing".

In this framework, $c_{\chi_{\alpha}}$ has the form

$$
c_{\chi_{\alpha}}: P_{1} \rightarrow Q_{1} \rightarrow P_{2} \rightarrow \cdots \rightarrow P_{y} \rightarrow Q_{y} \rightarrow \cdots \rightarrow P_{z} \rightarrow Q_{z} \rightarrow \cdots \rightarrow P_{l} \rightarrow Q_{l}
$$

where the paths $P_{y}$ and $P_{z}$ in $G_{1}$ are separated into two halves as

$$
P_{y}: P_{y}^{(1)} \rightarrow v^{*} \rightarrow P_{y}^{(2)}
$$

and

$$
P_{z}: P_{z}^{(1)} \rightarrow v^{*} \rightarrow P_{z}^{(2)}
$$

Remember that $P_{y}$ and $P_{z}$ are paths that stop over at various vertices depending on the permutation $\chi_{\alpha}$. The vertex $v^{*}$ must have adjacent stop-over vertices in each of the two paths $P_{y}$ and $P_{z}$. Let the nearest stop-over vertices on the paths $P_{y}$ and $P_{z}$ be $v_{\varphi_{y}}$ and $v_{\varphi_{y+1}}$, and $v_{\varphi_{z}}$ and $v_{\varphi_{z+1}}$, respectively.

This implies $\chi_{\alpha}$ has the form

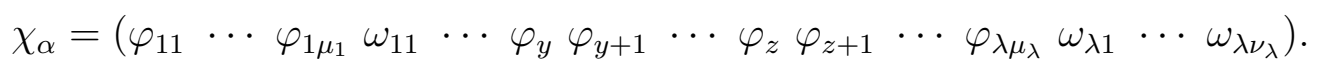


We can now precisely define the result of breaking. We define $\chi_{\beta}$ and $\chi_{\gamma}$ by splitting $\chi_{\alpha}$ as follows:

$$
\chi_{\beta}=\left(\varphi_{11} \cdots \varphi_{y} \varphi_{z+1} \cdots \omega_{\lambda \nu_{\lambda}}\right)
$$

and

$$
\chi_{\gamma}=\left(\varphi_{y+1} \cdots \varphi_{z}\right),
$$

with the necessary rewriting of $\chi_{\gamma}$ to have as its initial entry the value $\Phi\left(\chi_{\gamma}\right)$. Define $c_{\chi_{\beta}}$ and $c_{\chi_{\gamma}}$ to be

$$
c_{\chi_{\beta}}: P_{1} \rightarrow Q_{1} \rightarrow P_{2} \rightarrow \cdots \rightarrow P_{y}^{(1)} \rightarrow v^{*} \rightarrow P_{z}^{(2)} \rightarrow Q_{z} \rightarrow \cdots \rightarrow P_{l} \rightarrow Q_{l},
$$

and

$$
c_{\chi_{\gamma}}: P_{z}^{(1)} \rightarrow v^{*} \rightarrow P_{y}^{(2)} \rightarrow Q_{y} \rightarrow \cdots \rightarrow Q_{z-1},
$$

again changing the starting vertex of $c_{\chi_{\gamma}}$ to $v_{\Phi\left(c_{\chi \gamma}\right)}$.

We define the breaking of the WSP-pair with the above inputs to be the WSP-pair $\left(\mathcal{W}^{\prime}, \sigma^{\prime}\right)$ such that

$$
\mathcal{W}^{\prime}=\mathcal{W} \cup\left\{c_{\chi_{\beta}}, c_{\chi_{\gamma}}\right\} \backslash\left\{c_{\chi_{\alpha}}\right\}
$$

and

$$
\sigma^{\prime}=\sigma \chi_{\alpha}^{-1} \chi_{\beta} \chi_{\gamma}=\sigma \cdot\left(\varphi_{y+1} \varphi_{z+1}\right)
$$

The edge set of $\mathcal{W}$ is equal to the edge set of $\mathcal{W}^{\prime}$, so the weight of the modified cycle systems is the same as the original. Since we changed $\sigma$ to $\sigma^{\prime}$ by multiplying only by a transposition, the sign of the modified WSP-pair is opposite to that of the original.

By only discussing the case when $v^{*}$ is not distinguished, we avoid notational issues brought upon by cases when $v^{*}$ is or is not one of the stop-over vertices.

\subsection{Proof of Lemma 2.1, Part II}

Having defined breaking and sewing, we can continue the proof.

For any WSP-pair $(\mathcal{W}, \sigma)$ satisfying either Property 1 or Property 2, let $c_{\chi_{\alpha}} \in \mathcal{W}$ be the first walk in the initial term order with an vertex of intersection. Let $v^{*}$ be the first vertex of intersection in $c_{\chi_{\alpha}}$. Then for all walks $c$ with one or more self-intersections at $v^{*}$, continue to break $c$ at $v^{*}$ until there are no more self-intersections. Define the resulting WSP-pair $\left(\mathcal{W}_{u}, \sigma_{u}\right)$ to be the unlinked WSP-pair associated to $(\mathcal{W}, \sigma)$. In $\left(\mathcal{W}_{u}, \sigma_{u}\right)$, there is some number $N$ of general walks intersecting at vertex $v^{*}$. There may be a 2-cycle intersecting $v^{*}$ as well, but this does not matter.

For any permutation $\xi \in S_{N}$, let $\xi=\zeta_{1} \zeta_{2} \cdots \zeta_{\eta}$ be its cycle representation, where each $\zeta_{\iota}$ is a cycle. For each $1 \leq \iota \leq \eta$, sew together walks in order: if $\zeta_{\iota}=\left(\delta_{\iota 1} \cdots \delta_{\iota \varepsilon_{\iota}}\right)$, sew together $c_{\chi_{\delta_{t 1}}}$ and $c_{\chi_{\delta_{t 2}}}$ at $v^{*}$. Sew this result together with $c_{\chi_{\delta_{\iota 3}}}$, and so on through $c_{\chi_{\delta_{t \varepsilon}}}$. Note that the result of these sewings is unique, and that every WSP-pair $(\mathcal{W}, \sigma)$ with $\left(\mathcal{W}_{u}, \sigma_{u}\right)$ as its unlinked WSP-pair can be obtained in this way, and no other WSP-pair appears. We can perform this procedure for any $\xi \in S_{N}$; the sign of the resulting walk 
system $\left(C_{\xi}, \sigma_{\xi}\right)$ is $\operatorname{sgn}\left(C_{\xi}, \sigma_{\xi}\right)=\operatorname{sgn}(\xi) \operatorname{sgn}\left(\mathcal{W}_{u}, \sigma_{u}\right)$. This means that the contribution to the determinant of the weights of all WSP-pairs in the family $\mathcal{F}$ is

$$
\sum_{\xi \in S_{N}} \operatorname{sgn}(\xi) \operatorname{sgn}\left(\mathcal{W}_{u}, \sigma_{u}\right) \operatorname{wt}\left(\mathcal{W}_{u}, \sigma_{u}\right)=\operatorname{sgn}\left(\mathcal{W}_{u}, \sigma_{u}\right) \operatorname{wt}\left(\mathcal{W}_{u}, \sigma_{u}\right) \sum_{\xi \in S_{N}} \operatorname{sgn}(\xi)=0
$$

So elements of the same family cancel out in the determinant of $M_{H}$, giving that the contributions of all WSP-pairs satisfying either Property 1 or Property 2 cancel out in the hamburger determinant.

\subsection{Proof of Lemma 2.2}

Recall Properties 3 and 4 as well as Lemma 2.2.

Property 3. The walk system has two intersecting walks, one of which is a 2-cycle.

Property 4. The walk system is not minimal.

Lemma 2.2. The set of all walk systems $\mathcal{W}$ that satisfy neither Property 1 nor Property 2 and that satisfy Property 3 or Property 4 can be partitioned into equivalence classes, each of which contributes a net zero to the permutation expansion of the determinant of $M_{H}$.

In the proof of Lemma 2.2, we do not base our family $\mathcal{F}$ around a singular vertex; instead, we find a set of violations that each member of the family has. If the WSP-pair satisfies the hypotheses of Lemma 2.2, then either there is some 2-cycle $c: v_{\varphi} \rightarrow w_{\omega} \rightarrow v_{\varphi}$ that intersects with some other walk or there is some non-minimal permutation cycle.

Define a set of indices $I \subseteq[k]$ of violations, of which an integer can become a member in one of two ways. If $(\mathcal{W}, \sigma)$ is not minimal, there is at least one permutation cycle $\chi_{\alpha}$ with more than two consecutive $\varphi$ 's or $\omega$ 's in its cycle notation. For any intermediary $\iota$ between two $\varphi$ 's or $\iota+k$ between two $\omega$ 's, place $\iota$ in $I$. For example, if $\chi_{\alpha}=\left(\cdots \varphi^{\prime} \iota \varphi^{\prime \prime} \cdots\right)$, we place $\iota \in I$. Alternatively, there may be a 2-cycle $c: v_{i} \rightarrow w_{k+i} \rightarrow v_{i}$ such that either $v_{i}$ is a vertex in some other walk $c_{\chi_{\beta}}$ or $w_{k+i}$ is a vertex in some other cycle $c_{\chi_{\gamma}}$, or both. We declare this $i$ to be in $I$ as well.

Note that any WSP-pair $(\mathcal{W}, \sigma)$ satisfying either Property 3 or Property 4 has a nonempty set $I$. From our original WSP-pair, obtain a minimal WSP-pair $\left(\mathcal{W}_{m}, \sigma_{m}\right)$ by removing any transposition $\chi_{\alpha}$ from $\sigma$ and its corresponding 2-cycle $c_{\chi_{\alpha}}$ from $\mathcal{W}$, and also for any non-minimal permutation cycle $\chi_{\beta}$ we remove any intermediary $\varphi$ 's or $\omega$ 's from $\sigma$. We do not change the associated walk $c_{\chi_{\beta}}$ in $\mathcal{W}$ since it still corresponds to this minimized permutation cycle.

Let $i$ be any element in $I$. Since $i \in I$, the 2-cycle $c_{i}: v_{i} \rightarrow w_{k+i} \rightarrow v_{i}$ intersects some walk of $\mathcal{W}_{m}$ either at $v_{i}$, at $w_{k+i}$, or both. So there are four cases:

Case 1. $c_{i}$ intersects a walk $c_{\chi_{\beta}}$ at $v_{i}$ and no walk at $w_{k+i}$.

Case 2. $c_{i}$ intersects a walk $c_{\chi_{\gamma}}$ at $w_{k+i}$ and no walk at $v_{i}$.

Case 3. $c_{i}$ intersects a walk $c_{\chi_{\beta}}$ at $v_{i}$ and the same walk again at $w_{k+i}$. 

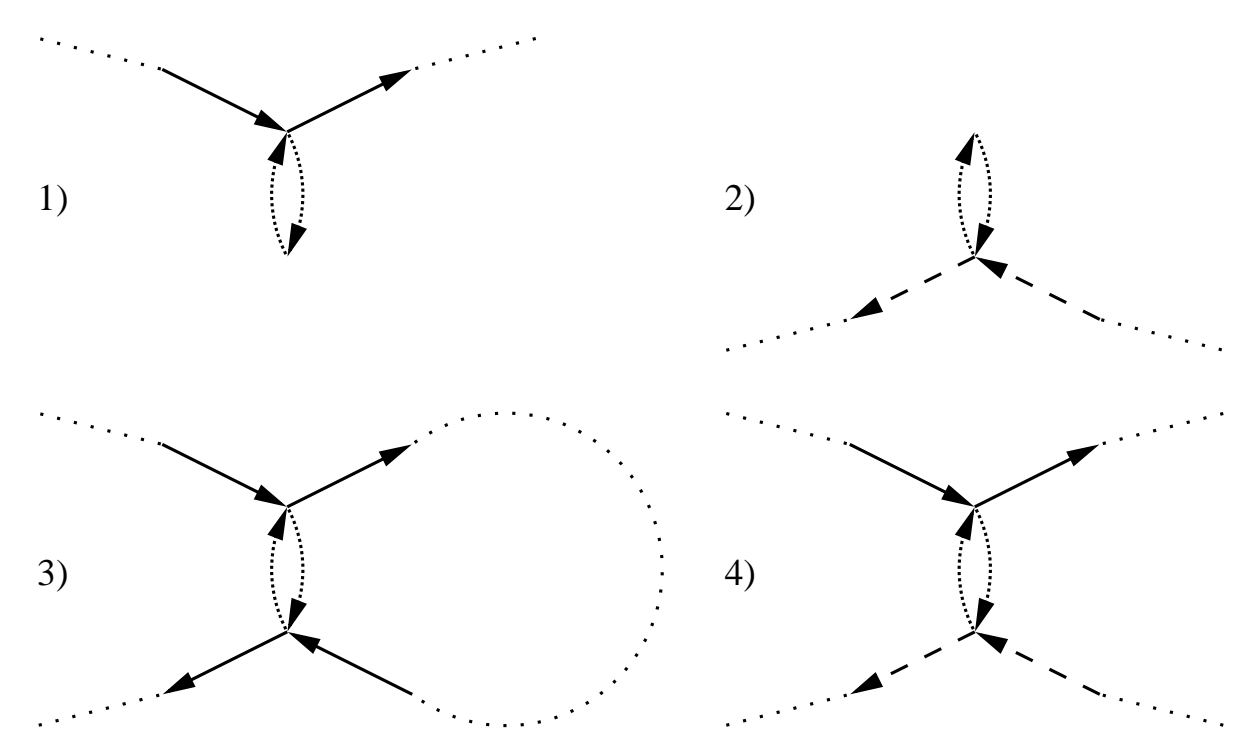

Figure 7: The four cases in which a walk can intersect with a 2-cycle

Case 4. $c_{i}$ intersects a walk $c_{\chi_{\beta}}$ at $v_{i}$ and another walk $c_{\chi_{\gamma}}$ at $w_{k+i}$.

See Figure 7 for a picture.

In order to build the family $\mathcal{F}$, we modify the minimal WSP-pair $\left(\mathcal{W}_{m}, \sigma_{m}\right)$ at each $i$ with some choice of options, each giving a possible WSP-pair that has $\left(\mathcal{W}_{m}, \sigma_{m}\right)$ as its minimal WSP-pair. We define a set of two or four options $\mathcal{O}_{i}$ for each $i$.

In Case 1, there are two options. Let $o_{1}$ be the option of including the walk $c_{i}$ in $\mathcal{W}_{m}$ and its corresponding transposition $\chi_{\alpha}$ in $\sigma_{m}$. Let $o_{2}$ be the option of including the intermediary $\varphi=i$ in $\chi_{\beta}$ in the position where $c_{\chi_{\beta}}$ passes through $v_{i}$.

[Note that we could not apply both options at the same time since the resulting $\chi_{\alpha}$ and $\chi_{\beta}$ would not be disjoint and therefore is not a term in the permutation expansion of the determinant.]

Similarly in Case 2, there are two options. Let $o_{1}$ be the option of including the walk $c_{i}$ in $\mathcal{W}_{m}$ and its corresponding transposition $\chi_{\alpha}$ in $\sigma_{m}$. Let $o_{2}$ be the option of including the intermediary $\omega=i+k$ in $\chi_{\gamma}$ in the position where $c_{\chi_{\gamma}}$ passes through $w_{k+i}$.

In Case 3, there are four options. Let $o_{1}$ be the option of including the walk $c_{i}$ in $\mathcal{W}_{m}$ and its corresponding transposition $\chi_{\alpha}$ in $\sigma_{m}$. Let $o_{2}$ be the option of including the intermediary $\varphi=i$ in $\chi_{\beta}$ in the position where $c_{\chi_{\beta}}$ passes through $v_{i}$. Let $o_{3}$ be the option of including the intermediary $\omega=i+k$ in $\chi_{\beta}$ in the position where $c_{\chi_{\beta}}$ passes through $w_{k+i}$. Let $o_{4}$ be the option of including both intermediaries $\varphi=i$ and $\omega=i+k$ in $\chi_{\beta}$ in the respective positions where $c_{\chi_{\beta}}$ passes through $v_{i}$ and $w_{k+i}$.

In Case 4 , there are four options. Let $o_{1}$ be the option of including the walk $c_{i}$ in $\mathcal{W}_{m}$ and its corresponding transposition $\chi_{\alpha}$ in $\sigma_{m}$. Let $o_{2}$ be the option of including the intermediary $\varphi=i$ in $\chi_{\beta}$ in the position where $c_{\chi_{\beta}}$ passes through $v_{i}$. Let $o_{3}$ be the option of including the intermediary $\omega=i+k$ in $\chi_{\gamma}$ in the position where $c_{\chi_{\gamma}}$ passes through $w_{k+i}$. Let $o_{4}$ be the option of including intermediary $\varphi=i$ in $\chi_{\beta}$ in the position where 


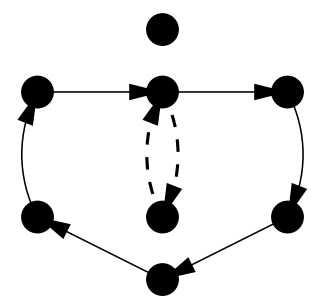

$(1364)(25)$

$\operatorname{sign}=+1$

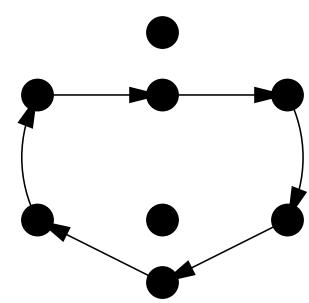

(12364)

sign $=-1$

Figure 8: A family of two walk systems that cancel in the hamburger determinant

$c_{\chi_{\beta}}$ passes through $v_{i}$ and intermediary $\omega=i+k$ in $\chi_{\gamma}$ in the position where $c_{\chi_{\gamma}}$ passes through $w_{k+i}$.

Corresponding to the associated minimal WSP-pair $\left(\mathcal{W}_{m}, \sigma_{m}\right)$ and index set $I$, define the family $\mathcal{F}$ to be the set of WSP-pairs $\left(\mathcal{W}_{f}, \sigma_{f}\right)$ by exercising all combinations of options $o_{j_{i}} \in \mathcal{O}_{i}$ on $\left(\mathcal{W}_{m}, \sigma_{m}\right)$ for each $i \in I$. Note that every WSP-pair derived in this fashion is a WSP-pair satisfying the hypotheses of Lemma 2.2 and is such that its associated minimal WSP-pair is $\left(\mathcal{W}_{m}, \sigma_{m}\right)$. There is also no other WSP-pair $\left(\mathcal{W}^{\prime}, \sigma^{\prime}\right)$ with $\left(\mathcal{W}_{m}, \sigma_{m}\right)$ as its minimal WSP-pair. See Figure 8 for a canceling family of two walk systems, one of which is the non-minimal walk system from Figure 5.

Every WSP-pair in $\mathcal{F}$ has the same weight since each option changes the edge set of $\mathcal{W}$ by at most a 2-cycle $v_{i} \rightarrow w_{k+i} \rightarrow v_{i}$, where $2 \leq i \leq k-1$ and each of those 2-cycles contributes a multiplier of 1 to the weight of the WSP-pair. The peculiar bounds in this restriction are due to the structure of the hamburger graph. A walk through the vertices $v_{1}$ and $w_{k+1}$ (or $v_{k}$ and $w_{2 k}$ ) must use edge $e_{1}^{\prime}$ (or $e_{k}$ ), and include in its associated permutation cycle 1 and $k+1$ (or $k$ and $2 k$ ). This implies that we can not have multiple walks through any of these four special vertices, as that would yield two non-disjoint permutation cycles in $\sigma$.

The sign of every WSP-pair in $\mathcal{F}$ is determined by the set of options applied to $\left(\mathcal{W}_{m}, \sigma_{m}\right)$. Each option $o_{j}$ contributes a multiplicative \pm 1 depending on $j-\operatorname{sgn}\left(o_{1}\right)=+1$, $\operatorname{sgn}\left(o_{2}\right)=-1$, and $\operatorname{sgn}\left(o_{3}\right)=-1$ and $\operatorname{sgn}\left(o_{4}\right)=+1$ if they exist.

Each family $\mathcal{F}$ contributes a cumulative weight of 0 . This is because there are the same number of positive $\left(\mathcal{W}_{f}, \sigma_{f}\right) \in \mathcal{F}$ as negative $\left(\mathcal{W}_{f}, \sigma_{f}\right) \in F$. We see this since if $i^{*} \in I$ then any set of WSP-pairs that exercise the same options $o_{j_{i}}$ for all $i \neq i^{*}$ has either two or four members (depending on the case of $i^{*}$ ), which split evenly between positive and negative WSP-pairs. Since each family contributes a net zero to the hamburger determinant, the total contribution from WSP-pairs satisfying the hypotheses of Lemma 2.2 is zero.

Since every WSP-pair appears once in the permutation expansion of $\operatorname{det} M_{H}$, the only WSP-pairs that contribute to the sum are those that are simple and minimal. Therefore $\operatorname{det} M_{H}$ is the sum over such WSP-pairs of $\operatorname{sgn}(\mathcal{W}, \sigma) \cdot \operatorname{wt}(\mathcal{W}, \sigma)$. This establishes Theorem 2.3 and gives Theorem 1.1 as a special case. 

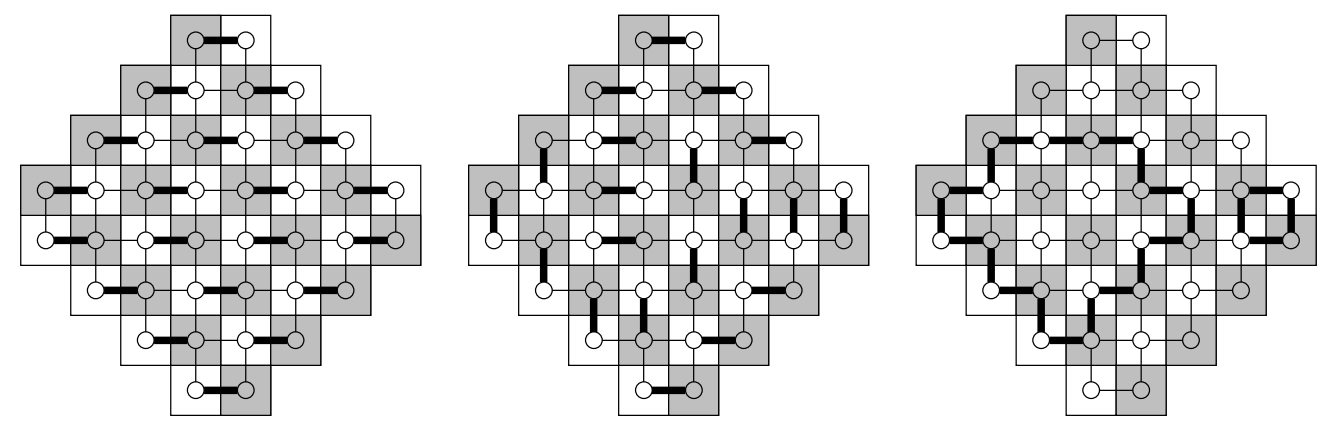

Figure 9: The symmetric difference of two matchings gives a cycle system

\section{Applications of the Hamburger Theorem}

We discuss first the application of the Hamburger Theorem in the case where the region is an Aztec diamond, mirroring results of Brualdi and Kirkland. Then we discuss the results from the case where the region is an Aztec pillow, and along the way, we explain how to implement the Hamburger Theorem when our region is any generalized Aztec pillow.

\subsection{Domino Tilings and Digraphs}

The Hamburger Theorem applies to counting the number of domino tilings of Aztec diamonds and generalized Aztec pillows. To illustrate this connection, we count domino tilings of the Aztec diamond by counting an equivalent quantity, the number of matchings on the dual graph $G$ of the Aztec diamond. We use the natural matching $N$ of horizontal neighbors in $G$, as exemplified in Figure 9 a on $A D_{4}$, as a reference point. Given any other matching $M$ on $G$, such as in Figure 9b, its symmetric difference with the natural matching is a collection of cycles in the graph, such as in Figure 9c.

When we orient the edges in $N$ from black vertices to white vertices and in $M$ from white vertices to black vertices, the symmetric difference becomes a collection of directed cycles. Notice that edges in the upper half of $G$ all go from left to right and the edges in the bottom half of $G$ go from right to left.

Since the edges of $N$ always appear in the cycles, we can contract the edges of $N$ to vertices of a new graph which retains its structure in terms of the cycle systems it produces. This new graph $H$ is of the form in Figure 10a. This argument shows that the number of domino tilings of an Aztec diamond equals the number of cycle systems of this new condensed graph, called the region's digraph.

We wish to concretize this notion of a digraph of the Aztec diamond $A D_{n}$. Given the natural tiling of an Aztec diamond consisting solely of horizontal dominoes, we place a vertex in the center of every domino. The edges of this digraph fall into three families. From every vertex in the top half of the diamond, create edges to the east, to the northeast, and to the southeast whenever there is a vertex there. From every vertex in the bottom half of the diamond, form edges to the west, to the southwest, and to the northwest 

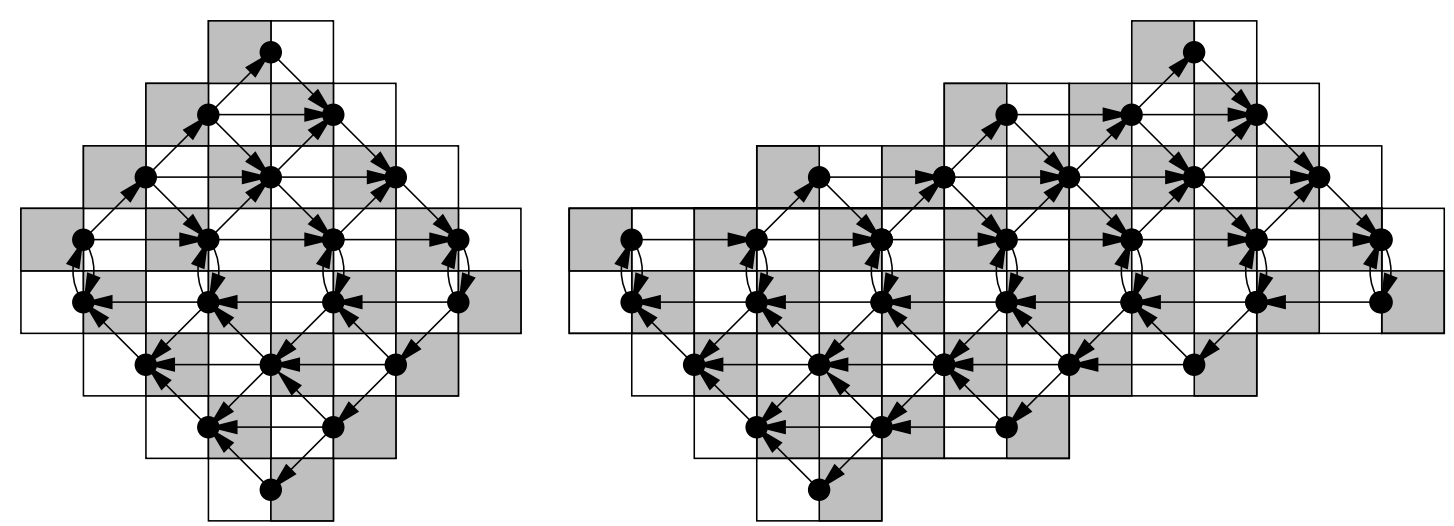

Figure 10: The hamburger graph for an Aztec diamond and an Aztec pillow

whenever there is a vertex there. Additionally, label the bottom vertices in the top half $v_{1}$ through $v_{n}$ from west to east and the top vertices in the bottom half $w_{n+1}$ through $w_{2 n}$, also west to east. For all $i$ between 1 and $n$, create a directed edge from $v_{i}$ to $w_{n+i}$ and from $w_{n+i}$ to $v_{i}$. The result when this construction is applied to $A D_{4}$ is a graph of the form in Figure 10a. By construction, this graph is a hamburger graph.

Theorem 5.1. The digraph of an Aztec diamond is a hamburger graph.

Since both the upper half of the digraph and the lower half of the digraph are strongly planar, there are no negative cycle systems. This implies that the determinant of the corresponding hamburger matrix counts exactly the number of cycle systems in the digraph.

Corollary 5.2. The number of domino tilings of an Aztec diamond is the determinant of its hamburger matrix.

\subsection{Applying the Hamburger Theorem to Aztec Diamonds}

In order to apply Theorem 1.1 to count the number of tilings of $A D_{n}$, we need to find the number of paths in the upper half of $D$ from $v_{i}$ to $v_{j}$ and the number of paths in the lower half of $D$ from $w_{n+j}$ to $w_{n+i}$. The key observation is that by the equivalence in Figure 11, we are in effect counting the number of paths from $(i, i)$ to $(j, j)$ using steps of size $(0,1),(1,0)$, or $(1,1)$ that do not pass above the line $y=x$. This is a combinatorial interpretation for the $(j-i)$-th large Schröder number. The large Schröder numbers $s_{0}, \ldots, s_{5}$ are $1,2,6,22,90,394$. This is sequence number A006318 in the Encyclopedia of Integer Sequences [13].

Corollary 5.3. The number of domino tilings of the Aztec diamond $A D_{n}$ is equal to

$$
\# A D_{n}=\operatorname{det}\left[\begin{array}{cc}
S_{n} & I_{n} \\
-I_{n} & S_{n}^{T}
\end{array}\right]
$$

where $S_{n}$ is an upper triangular matrix with the $(i, j)$-th entry equal to $s_{j-i}$ for $j \geq i$. 

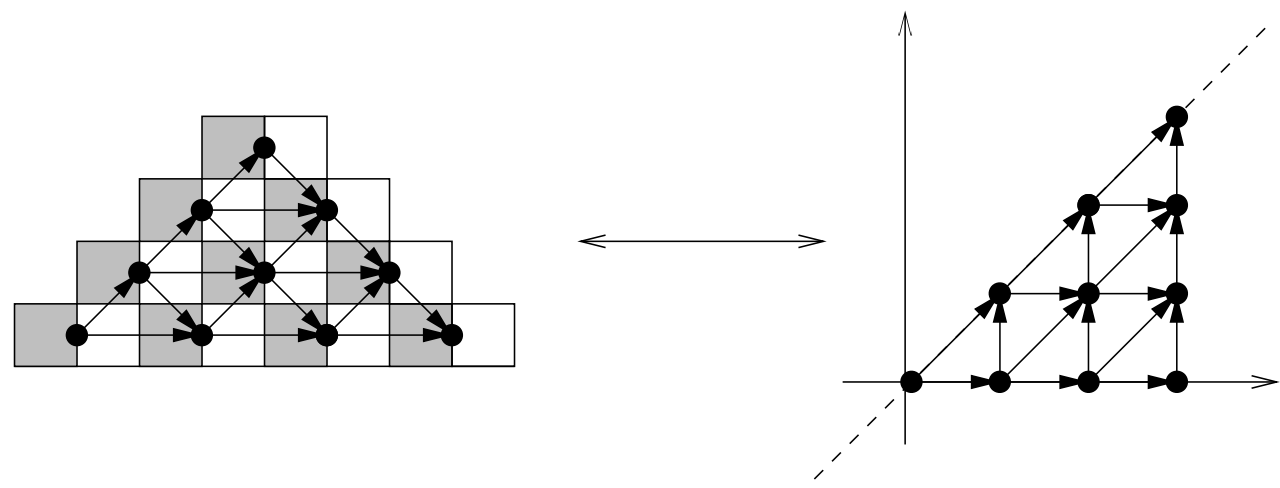

Figure 11: The equivalence between paths in $D$ and lattice paths in the first quadrant

For example, when $n=6$, the matrix $S_{6}$ is

$$
S_{6}=\left[\begin{array}{cccccc}
1 & 2 & 6 & 22 & 90 & 394 \\
0 & 1 & 2 & 6 & 22 & 90 \\
0 & 0 & 1 & 2 & 6 & 22 \\
0 & 0 & 0 & 1 & 2 & 6 \\
0 & 0 & 0 & 0 & 1 & 2 \\
0 & 0 & 0 & 0 & 0 & 1
\end{array}\right] .
$$

Brualdi and Kirkland prove a similar determinant formula for the number of tilings of an Aztec diamond in a matrix-theoretical fashion based on the $n(n+1) \times n(n+1)$ Kasteleyn matrix of the graph $H$ and a Schur complement calculation. The Hamburger Theorem gives a purely combinatorial way to reduce the calculation of the number of tilings of an Aztec diamond to the calculation of a $2 n \times 2 n$ Hamburger determinant.

Following cues from Brualdi and Kirkland, we can reduce this to an $n \times n$ determinant via a Schur complement. For uses and the history of the Schur Complement, see the new book [14] by Zhang. In the case of the block matrix $M_{H}$ in Equation (1), taking the Schur complement of $B$ in $M_{H}$ gives

$$
\begin{aligned}
\operatorname{det} M_{H} & =\operatorname{det}\left(\begin{array}{cc}
A & D_{1} \\
-D_{2} & B
\end{array}\right) \cdot \operatorname{det}\left(\begin{array}{cc}
I & 0 \\
B^{-1} D_{2} & I
\end{array}\right) \\
& =\operatorname{det}\left(\begin{array}{cc}
A+D_{1} B^{-1} D_{2} & D_{1} \\
0 & B
\end{array}\right) \\
& =\operatorname{det}\left(A+D_{1} B^{-1} D_{2}\right) \cdot \operatorname{det} B \\
& =\operatorname{det}\left(A+D_{1} B^{-1} D_{2}\right),
\end{aligned}
$$

since $B$ is a lower triangular matrix with 1's on the diagonal. In this way, every hamburger determinant can be reduced to a smaller determinant of a Schur complement matrix. In the case of a simple hamburger graph where $D_{2}=D_{1}=I$, the determinant reduces further to $\operatorname{det}\left(A+B^{-1}\right)$. Lastly, in the case where the hamburger graph is rotationally 
symmetric, $B=J A J$ where $J$ is the exchange matrix, which consists of 1 's down the main skew-diagonal and 0's elsewhere. This implies that we can write the determinant in terms of just the submatrix $A$, i.e., $\operatorname{det}\left(A+J A^{-1} J\right)$. (Note that $J^{-1}=J$.)

Corollary 5.4. The number of domino tilings of the Aztec diamond $A D_{n}$ is equal to $\operatorname{det}\left(S_{n}+J_{n} S_{n}^{-1} J_{n}\right)$, where $J_{n}$ is the $n \times n$ exchange matrix.

In the case of a hamburger graph $H$, we call this Schur complement, $A+B^{-1}$, a reduced hamburger matrix. In the Aztec diamond graph example above, we can thus calculate the number of tilings of the Aztec diamond $A D_{6}$ as follows. The inverse of $S_{6}$ is

$$
S_{6}^{-1}=\left[\begin{array}{rrrrrr}
1 & -2 & -2 & -6 & -22 & -90 \\
0 & 1 & -2 & -2 & -6 & -22 \\
0 & 0 & 1 & -2 & -2 & -6 \\
0 & 0 & 0 & 1 & -2 & -2 \\
0 & 0 & 0 & 0 & 1 & -2 \\
0 & 0 & 0 & 0 & 0 & 1
\end{array}\right]
$$

which implies that the determinant of the reduced hamburger matrix

$$
M_{6}=\left[\begin{array}{rrrrrr}
2 & 2 & 6 & 22 & 90 & 394 \\
-2 & 2 & 2 & 6 & 22 & 90 \\
-2 & -2 & 2 & 2 & 6 & 22 \\
-6 & -2 & -2 & 2 & 2 & 6 \\
-22 & -6 & -2 & -2 & 2 & 2 \\
-90 & -22 & -6 & -2 & -2 & 2
\end{array}\right]
$$

gives the number of tilings of $A D_{6}$.

Brualdi and Kirkland were the first to find such a determinantal formula for the number of tilings of an Aztec diamond [2]. Their matrix was different only in the fact that each entry was multiplied by $(-1)$ and there was a multiplicative factor of $(-1)^{n}$. Brualdi and Kirkland were able to calculate the sequence of determinants, $\left\{\operatorname{det} M_{n}\right\}$, using a $J$-fraction expansion, which only works when matrices are Toeplitz or Hankel.

$\mathrm{Eu}$ and $\mathrm{Fu}$ also found an $n \times n$ determinant that calculated the number of tilings of an Aztec diamond [3]. Their matrix also involves large Schröder numbers; when $n=6$, their matrix is

$$
\left[\begin{array}{rrrrrr}
1 & 2 & 6 & 22 & 90 & 394 \\
2 & 6 & 22 & 90 & 394 & 1806 \\
6 & 22 & 90 & 394 & 1806 & 8558 \\
22 & 90 & 394 & 1806 & 8558 & 41586 \\
90 & 394 & 1806 & 8558 & 41586 & 206098 \\
394 & 1806 & 8558 & 41586 & 206098 & 1037718
\end{array}\right] .
$$

Their proof hinges on the relationship between domino tilings in an Aztec diamond and path systems in a lattice derived from the Aztec diamond's structure. The key observation is that these path systems in the lattice can be extended uniquely outside the Aztec 


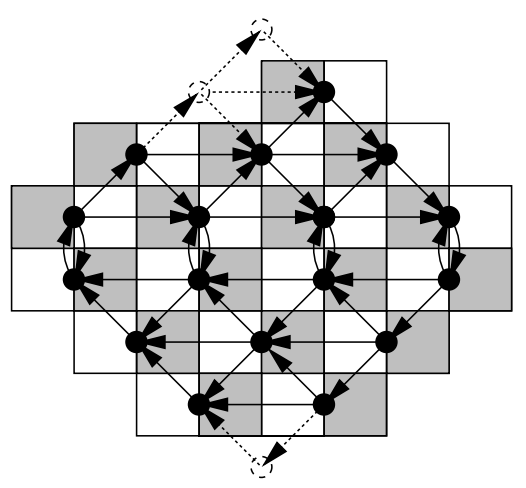

Figure 12: The digraph of a generalized Aztec pillow from the digraph of an Aztec diamond

diamond to form Schröder paths. Applying the Gessel-Viennot method gives the above matrix of Schröder numbers. Unfortunately, Eu and Fu's method does not generalize further, as explained at the end of the next section.

\subsection{Applying the Hamburger Theorem to Aztec Pillows}

A generalized Aztec pillow can be produced by restricting the placement of certain dominoes in an Aztec diamond in order to generate the desired boundary. We define the digraph of a generalized Aztec pillow to be the restriction of the digraph of an Aztec diamond to the vertices on the interior of the pillow. An example is presented Figure 12. Since the generalized Aztec pillow's digraph is a restriction of the Aztec diamond's digraph, we have the following corollary.

Corollary 5.5. The digraph of an Aztec pillow or a generalized Aztec pillow is a hamburger graph.

Aztec pillows were introduced in part because of an intriguing conjecture for the number of their tilings given by Propp [12].

Conjecture 5.6 (Propp's Conjecture). The number of tilings of an Aztec pillow $A P_{n}$ is a larger number squared times a smaller number. We write $\# A P_{n}=l_{n}^{2} s_{n}$. In addition, depending on the parity of $n$, the smaller number $s_{n}$ satisfies a simple generating function. For $A P_{2 m}$, the generating function is

$$
\sum_{m=0}^{\infty} s_{2 m} x^{m}=\left(1+3 x+x^{2}-x^{3}\right) /\left(1-2 x-2 x^{2}-2 x^{3}+x^{4}\right) .
$$

For $A P_{2 m+1}$, the generating function is

$$
\sum_{m=0}^{\infty} s_{2 m+1} x^{m}=\left(2+x+2 x^{2}-x^{3}\right) /\left(1-2 x-2 x^{2}-2 x^{3}+x^{4}\right) .
$$



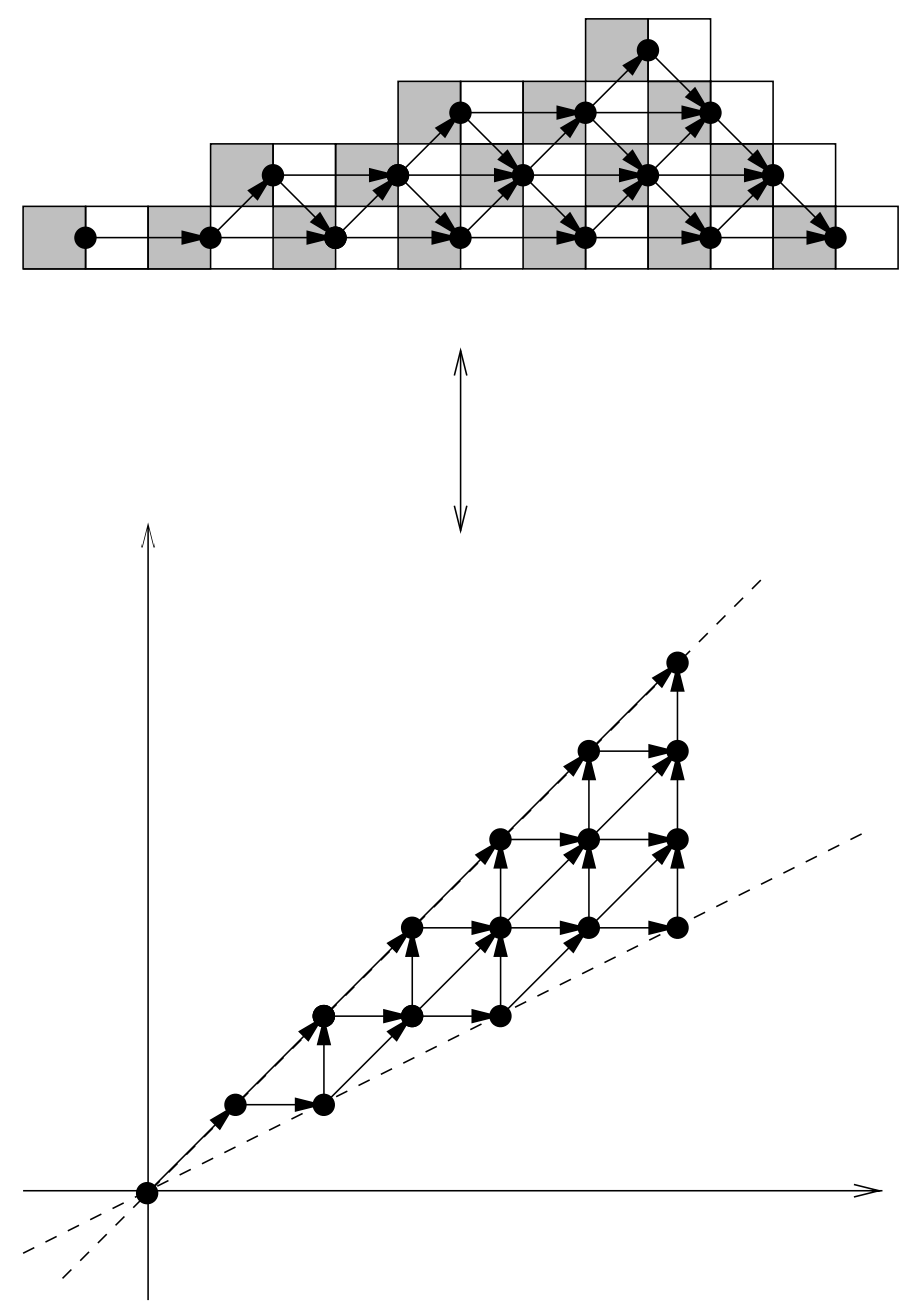

Figure 13: The equivalence between paths in $D$ and lattice paths in the first quadrant

My hope for the Hamburger Theorem was that it would give a proof of Propp's Conjecture. We shall see that although we achieve a faster determinantal method to calculate $\# A P_{n}$, the sequence of determinants is not calculable by a $J$-factor expansion, as was the case in Brualdi and Kirkland's work.

Using the same method as for Aztec diamonds, creating the hamburger graph $H$ for an Aztec pillow gives Figure 10b. Counting the number of paths from $v_{i}$ to $v_{j}$ and from $w_{n+j}$ to $w_{n+i}$ in successively larger Aztec pillows gives us the infinite upper-triangular array $\widetilde{S}=\left(\widetilde{s}_{i, j}\right)$ of modified Schröder numbers defined by the following combinatorial interpretation. Let $\widetilde{s}_{i, j}$ be the number of paths from $(i, i)$ to $(j, j)$ using steps of size $(0,1),(1,0)$, and $(1,1)$, not passing above the line $y=x$ nor below the line $y=x / 2$. The equivalence between these paths and paths in the upper half of the hamburger graph is shown in Figure 13. The principal $7 \times 7$ minor matrix $\widetilde{S}_{7}$ of $\widetilde{S}$ is 


$$
\widetilde{S}_{7}=\left[\begin{array}{rrrrrrr}
1 & 1 & 2 & 5 & 16 & 57 & 224 \\
0 & 1 & 2 & 5 & 16 & 57 & 224 \\
0 & 0 & 1 & 2 & 6 & 21 & 82 \\
0 & 0 & 0 & 1 & 2 & 6 & 22 \\
0 & 0 & 0 & 0 & 1 & 2 & 6 \\
0 & 0 & 0 & 0 & 0 & 1 & 2 \\
0 & 0 & 0 & 0 & 0 & 0 & 1
\end{array}\right]
$$

This table of numbers is sequence number A114292 in the Encyclopedia of Integer Sequences [13].

Theorem 5.7. The number of domino tilings of an Aztec pillow of order $n$ is equal to

$$
\# A P_{n}=\operatorname{det}\left[\begin{array}{cc}
\widetilde{S}_{n} & I_{n} \\
-I_{n} & J_{n} \widetilde{S}_{n} J_{n}
\end{array}\right]
$$

where $\widetilde{S}_{n}$ is the $n \times n$ principal submatrix of $\widetilde{S}$ and $J_{n}$ is the $n \times n$ exchange matrix.

As in the case of Aztec diamonds, we can calculate the reduced hamburger matrix through a Schur calculation. The inverse of $\widetilde{S}_{6}$ is

$$
\widetilde{S}_{6}^{-1}=\left[\begin{array}{rrrrrr}
1 & -1 & 0 & 0 & 0 & 0 \\
0 & 1 & -2 & -1 & -2 & -5 \\
0 & 0 & 1 & -2 & -2 & -5 \\
0 & 0 & 0 & 1 & -2 & -2 \\
0 & 0 & 0 & 0 & 1 & -2 \\
0 & 0 & 0 & 0 & 0 & 1
\end{array}\right]
$$

and the resulting reduced hamburger matrix for $A P_{6}$ is

$$
M_{6}=\left[\begin{array}{rrrrrr}
2 & 1 & 2 & 5 & 16 & 57 \\
-2 & 2 & 2 & 5 & 16 & 57 \\
-2 & -2 & 2 & 2 & 6 & 21 \\
-5 & -2 & -2 & 2 & 2 & 6 \\
-5 & -2 & -1 & -2 & 2 & 2 \\
0 & 0 & 0 & 0 & -1 & 2
\end{array}\right] \text {. }
$$

This gives us a much faster way to calculate the number of domino tilings of an Aztec pillow than was known previously. We have reduced the calculation of the $O\left(n^{2}\right) \times O\left(n^{2}\right)$ Kasteleyn-Percus determinant to an $n \times n$ reduced hamburger matrix. To be fair, the Kasteleyn-Percus matrix has $-1,0$, and +1 entries while the reduced hamburger matrix may have very large entries, which makes theoretical running time comparisons difficult. Experimentally, when calculating the number of domino tilings of $A P_{14}$ using Maple 8.0 on a $447 \mathrm{MHz}$ Pentium III processor, the determinant of the $112 \times 112$ Kasteleyn-Percus matrix took 25.3 seconds while the determinant of the $14 \times 14$ reduced hamburger matrix took less than 0.1 seconds. 


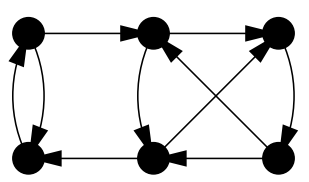

Figure 14: A simple generalized hamburger graph

Whereas we have a very understandable determinantal formula for the number of tilings of the region, this does not translate into a proof of Propp's Conjecture because we cannot calculate the determinant of the matrices $M_{n}$ explicitly. We cannot apply a $J$-fraction expansion as Brualdi and Kirkland did since the reduced hamburger matrix is not Toeplitz or Hankel. Also, we might hope to apply Eu and Fu's method. It is not hard to construct the background lattice derived from generalized Aztec pillows; however, the path systems that are associated to domino tilings of the region cannot be extended as $\mathrm{Eu}$ and $\mathrm{Fu}$ did. A Gessel-Viennot-derived matrix still exists, but its structure does not lend itself to a simple determinant calculation as in the case of Aztec diamonds.

\section{Generalizations}

In this section we present a counterexample to a natural generalization of the Hamburger Theorem and discuss an intriguing generalization of Propp's Conjecture.

\subsection{Counterexample to Generalizations of the Hamburger The- orem}

The structure of the hamburger graph presented in Figure 1 seems restrictive, so the question naturally arises whether it is somehow necessary. Can the edge set $E_{3}$ between graphs $G_{1}$ and $G_{2}$ be an arbitrary bipartite graph? The answer in general is no.

Take for example the simple graph $H$ in Figure 14. As one can count by hand, there are 10 distinct cycle systems in $H$, as enumerated in Figure 15. Creating the hamburger matrix that would correspond to this graph gives

$$
M_{H}=\left[\begin{array}{cccccc}
1 & 1 & 1 & 1 & 0 & 0 \\
0 & 1 & 1 & 0 & 1 & 0 \\
0 & 0 & 1 & 0 & 0 & 1 \\
-1 & 0 & 0 & 1 & 0 & 0 \\
0 & 0 & -1 & 1 & 1 & 0 \\
0 & -1 & 0 & 1 & 1 & 1
\end{array}\right]
$$

where the lower left block describes paths from $w_{4}, w_{5}$, and $w_{6}$ to $v_{1}, v_{2}$, and $v_{3}$ and we have changed no other block of the matrix. This seems the most logical extension of the hamburger matrix. 

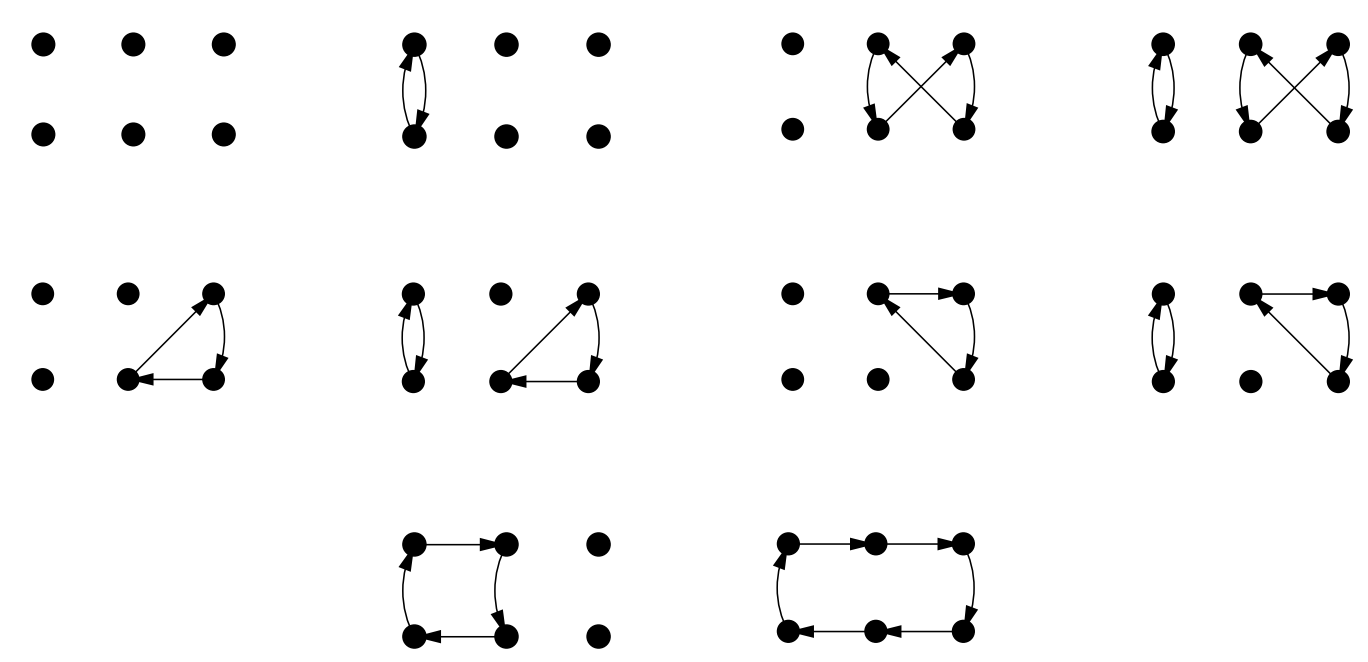

Figure 15: The ten cycle systems for the generalized hamburger graph in Figure 14

The determinant of this matrix is -5 . Even though one might expect to need a new sign convention on cycle systems in generalized hamburger graphs, any sign convention would necessarily conserve the parity of the number of cycle systems. Therefore there is no $+1 /-1$ labeling of the cycle systems in Figure 15 that would allow $\operatorname{det} M_{H}=\sum_{c \in \mathcal{C}} \operatorname{sgn}(c)$. This means that either the matrix for this generalized hamburger graph is not correct or that the theorem does not hold in general.

The only hope for my idea of how to generalize the hamburger matrix is what happens when one considers the difference between even and odd permutations. In the counterexample above, the edge set $E_{3}$ sends vertex $w_{4}$ to vertex $v_{1}, w_{5}$ to $v_{3}$, and $w_{6}$ to $v_{2}$. This can be thought of as an odd permutation of a hamburger's edge set (sending $w_{4}$ to $v_{1}, w_{5}$ to $v_{2}$, and $w_{6}$ to $v_{3}$ ). In my limited calculations so far, whenever the permutation of the natural edge set is even, the parity of the number of cycle systems, counted by hand, is equal to the parity of the determinant of the matrix. I have not found a sign convention for these graphs that explains the calculated determinant, but being equal in parity allows for hope of a possible extension to the Hamburger Theorem.

\subsection{Generalizing Propp's Conjecture}

A key motivation in the study of Aztec pillows is Propp's Conjecture for 3-pillows, presented in Section 5.3. Through experimental calculations, I have expanded and extended the conjecture.

For one, I extend the conjecture to odd pillows. For any odd number $q$, we can use the notation $A P_{n}^{q}$ to represent the $n$-th $q$-pillow for odd $q$. Recall from the introduction that this is a centrally symmetric region with steps of length $q$ and central belt of size $2 \times 2 n$. Calculating the number of tilings of $A P_{n}^{5}, A P_{n}^{7}$, and $A P_{n}^{9}$ for $n$ at least up to

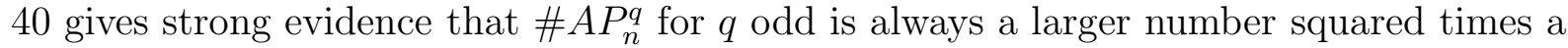
smaller number. As with 3-pillows, we can write $\# A P_{n}^{q}=l_{n, q}^{2} s_{n, q}$, where $s_{n, q}$ is relatively 

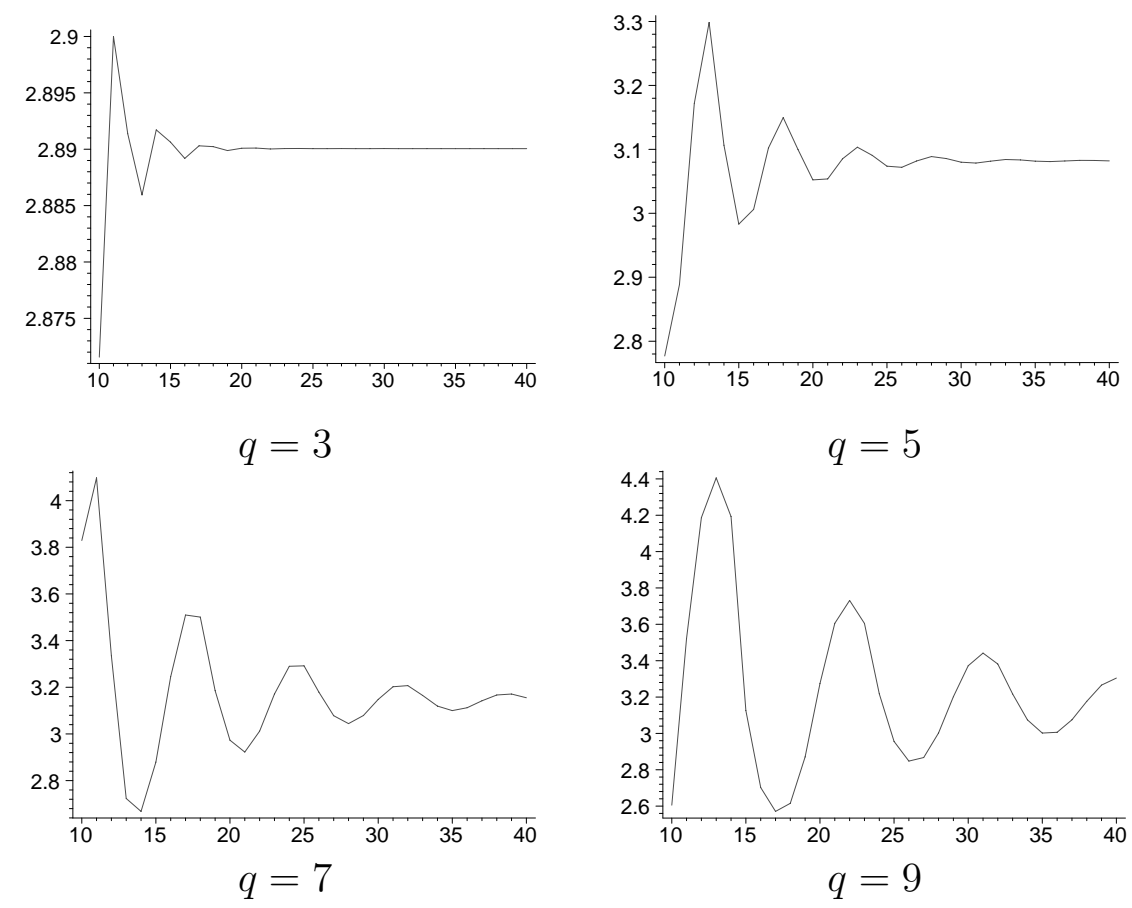

Figure 16: Plots of values of $s_{n, q} / s_{n-2, q}$ for $q=3,5,7,9$

small but is not necessarily the squarefree part of $\# A P_{n}^{q}$.

Additional structure in these smaller numbers also appears. Propp's Conjecture gives an explicit recurrence for the smaller-number values when $q=3$. In the case $q=5$, there is no linear, constant coefficient $k$-th degree recurrence for any $k$ up to 20 . However in each case $(q=5, q=7$, and $q=9)$ the structure is undeniable. Plotting the values of $s_{n, q} / s_{n-2, q}$ gives a remarkable damped sinusoidal graph converging to some number $\rho$. (See Figure 16.)

In addition, plotting the values of $s_{n, q} / s_{n-1, q}$ depending on whether $n$ is even or odd gives two damped sinusoidal graphs converging to some values $\tau$ and $2 \tau$ respectively. This implies $\rho=2 \tau^{2}$. Approximate values for $\rho$ are given in Table 1. We have included the values for Aztec diamonds $(q=1)$ and the original Aztec pillows $(q=3)$.

\begin{tabular}{ll}
$q$ & Approximate limit of $s_{n, q} / s_{n-2, q}$ \\
\hline 1 & 2 \\
3 & 2.890053636 \\
5 & 3.0821372 \\
7 & 3.145 \\
9 & 3.18
\end{tabular}

Table 1: $\rho$ values for various values of $q$

Even more appears to be true. By construction, the dual graphs to all odd pillows 
are 2-even-symmetric - that is, centrally symmetric so that antipodes are an even path length apart. Jockusch [7] proved that the number of matchings of such a graph $G$ is the sum of two squares. In terms of the underlying graph, these two squares are the real and imaginary parts of a weighted matching of the graph $G_{2}$. If we consider $R_{2}$ to be the rotation by 180 degrees, the graph $G_{2}$ is derived from $G$ by taking a branch cut through the graph, removing the vertices on one side of the branch cut, and placing an new edge between any two vertices $v$ and $w$ such that $v$ and $R_{2}(w)$ were connected in $G$. The weighting gives these new edges the weight $i$ to the power of the net number of times the original edge in $G$ crossed the branch cut counterclockwise when traversing from the white vertex to the black vertex.

Through a different method in my doctoral dissertation [6], I also showed that the number of domino tilings of the region is a sum of two squares. This follows from the structure of the Kasteleyn-Percus matrix for a rotationally symmetric generalized Aztec pillow. After limited calculation, these two methods appear to give the same sums-ofsquares representation. For a $q$-pillow, we will call these values $a_{n, q}$ and $b_{n, q}$; that is, for any Aztec Pillow, we can write $\# A P_{n}^{q}=a_{n, q}^{2}+b_{n, q}^{2}$. Analysis of this set of squares yields unexpected information about the larger numbers $l_{n}$ appearing in Propp's Conjecture, and the larger numbers $l_{n, q}$ in general. For any odd pillow $A P_{n}^{q}, l_{n, q}$ divides both $a_{n, q}$ and $b_{n, q}$. This gives us new insight into the structure of the previously ununderstood $l_{n, q}$, and implies that $s_{n, q}$ is also a sum of squares. Thus, I formulate a new and improved conjecture about odd pillows.

Conjecture 6.1. The number of tilings of $A P_{n}^{q}$ is a larger number squared times a smaller number. There exist values $l_{n, q}$ and $s_{n, q}$ such that we can write $\# A P_{n}^{q}=l_{n, q}^{2} s_{n, q}$. The value $s_{n}$ satisfies the following structure. The ratio of $s_{2 n+1, q} / s_{2 n, q}$ to $s_{2 n+2, q} / s_{2 n+1, q}$ is

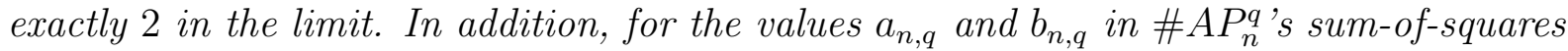
representation, $l_{n, q}$ divides both $a_{n, q}$ and $b_{n, q}$.

\subsection{Conclusion}

The Hamburger Theorem allows us to calculate the number of cycle systems in generalized Aztec Pillow graphs more quickly. It also allows us to count the number of cycle systems in many previously inaccessible graphs, such as non-planar hamburger graphs. Just as Gessel and Viennot's result has found many applications, I hope the Hamburger Theorem will be useful as well.

\subsection{Remarks}

This article is based on work in the author's doctoral dissertation [6]. A preliminary version of this article appeared as a poster at the 2005 International Conference on Formal Power Series and Algebraic Combinatorics in Taormina, Italy. The author would like to thank Henry Cohn and Tom Zaslavsky for numerous intriguing discussions and useful corrections. The author would also like to thank an anonymous referee for his or her time and suggestions for improving this article. 


\section{References}

[1] Martin Aigner. Lattice paths and determinants. In Computational Discrete Mathematics, volume 2122 of Lecture Notes in Comput. Sci., pages 1-12. Springer, Berlin, 2001.

[2] Richard Brualdi and Stephen Kirkland. Aztec diamonds and digraphs, and Hankel determinants of Schröder numbers. Submitted, 2003. Available at http://www.math.wisc.edu/ brualdi/aztec2.pdf.

[3] Sen-Peng Eu and Tung-Shan Fu. A simple proof of the Aztec diamond theorem. Electron. J. Combin., 12:Research Paper 18, 8 pp. (electronic), 2005. arXiv:math.CO/0412041.

[4] Ira Gessel and Xavier G. Viennot. Binomial determinants, paths, and hook length formulae. Adv. in Math., 58:300-321, 1985.

[5] Ira Gessel and Xavier G. Viennot. Determinants, paths, and plane partitions. Manuscript, 1989. Available at http://www.cs.brandeis.edu/ ira/papers/pp.pdf.

[6] Christopher R. H. Hanusa. A Gessel-Viennot-Type Method for Cycle Systems with Applications to Aztec Pillows, PhD Thesis, University of Washington, June 2005. Available at http://www.math.binghamton.edu/chanusa/papers/2005/Dissertation.pdf.

[7] W. Jockusch. Perfect matchings and perfect squares. J. Combin. Theory Ser. A, 67(1):100-115, 1994.

[8] Samuel Karlin and James McGregor. Coincidence probabilities. Pacific J. Math., 9:1141-1164, 1959.

[9] P. W. Kasteleyn. The statistics of dimers on a lattice I. The number of dimer arrangements on a quadratic lattice. Physica, 27:1209-1225, 1961.

[10] B. Lindström. On the vector representations of induced matroids. Bull. London Math. Soc., 5:85-90, 1973.

[11] J. Percus. One more technique for the dimer problem. J. Math. Phys., 10:1881-1884, 1969.

[12] James Propp. Enumeration of matchings: problems and progress. In New perspectives in algebraic combinatorics (Berkeley, CA, 1996-97), volume 38 of Math. Sci. Res. Inst. Publ., pages 255-291. Cambridge Univ. Press, Cambridge, 1999. arXiv:math.CO/9904150.

[13] N. J. A. Sloane. The On-Line Encyclopedia of Integer Sequences. http://www.research.att.com/ njas/sequences/.

[14] Fuzhen Zhang. The Schur Complement and its Applications, volume 4 of Numerical Methods and Algorithms. Springer, New York, 2005. 